\title{
Étude sur un monolithe - la dalle S12 au sol du monument de Gavrinis (Larmor-Baden, Morbihan)
}

Case study on a monolith - The paving stone S12 on the ground of Gavrinis monument (Larmor-Baden, Morbihan)

Die Untersuchung einer monolithischen Stele - Die Steinplatte S12 auf dem Boden des Grabmonumentes von Gavrinis (Larmor-Baden, Morbihan, Frankreich)

Serge Cassen, Valentin Grimaud, Laurent Lescop, Elven Robinet et Nancy Marcoux

\section{OpenEdition}

\section{Journals}

Édition électronique

URL : https://journals.openedition.org/rao/3284

DOI : $10.4000 /$ rao.3284

ISSN : 1775-3732

\section{Éditeur}

Presses universitaires de Rennes

\section{Édition imprimée}

Date de publication : 31 décembre 2016

Pagination : $55-76$

ISBN : 978-2-7535-5683-6

ISSN : 0767-709X

\section{Référence électronique}

Serge Cassen, Valentin Grimaud, Laurent Lescop, Elven Robinet et Nancy Marcoux, «Étude sur un monolithe - la dalle S12 au sol du monument de Gavrinis (Larmor-Baden, Morbihan) », Revue archéologique de l'Ouest [En ligne], 33 | 2016, mis en ligne le 31 juillet 2019, consulté le 23 août 2022 URL : http://journals.openedition.org/rao/3284 ; DOI : https://doi.org/10.4000/rao.3284 


\title{
Étude sur un monolithe - la dalle $S 12$ au sol du monument de Gavrinis (Larmor-Baden, Morbihan)
}

\author{
Case Study on a Monolith - The Paving Stone S12 on the Ground \\ of Gavrinis Monument (Larmor-Baden, Morbihan)
}

\author{
Serge Cassen ${ }^{\mathrm{a}}$, Valentin Grimaud ${ }^{\mathrm{b}}$, Laurent Lescop ${ }^{\mathrm{c}}$, \\ Elven Robinet ${ }^{\mathrm{d}}$ et Nancy Marcoux ${ }^{\mathrm{e}}$
}

\begin{abstract}
Résumé : À propos d'un monolithe extrait d'une des plus fameuses tombes à couloir néolithiques du territoire carnacéen, l'étude aborde des questions méthodologiques relatives à l'enregistrement des gravures, évidentes ou altérées, et des supports rocheux dans leur morphologie intime pouvant contribuer à la lecture et à l'interprétation des signes. Les différents outils informatiques employés sont testés et permettent un exercice comparatif entre tous les résultats obtenus, notamment en termes de dimensions, volumes et masses. Enfin, la conservation inattendue de restes carbonisés dans deux anfractuosités de la tranche fracturée a conduit à un essai de datation par le radiocarbone qui permet de préciser une chronologie absolue jusque-là déficiente pour ce monument.
\end{abstract}

\begin{abstract}
Dealing with a monolith extracted from one of the most famous Neolithic passage graves of the Carnacean area, this study approaches methodological issues concerning the recording of engravings, obvious or altered, and rocky supports in their intimate morphology that can contribute to the reading and interpretation of the signs. Different digital tools are tested, allowing a comparative exercise between all the results, particularly in terms of size, volumes and masses. Finally, the unexpected preservation of carbonized remains in crushed hollow of the broken edges led to a radiocarbon dating experiment that is included in an essay about the biography of an ancient stele.
\end{abstract}

Mots clés : Stèle en réemploi, Néolithique, lasergrammétrie, photogrammétrie, images compilées.

Keywords: Reused stele, Neolithic, lasergrammetry, photogrammetry, compiled images.

\section{LE CHOIX DE L'OBJET}

Après les premiers levés portant sur les figurations de lames de haches, menés en 2007 à Gavrinis (Morbihan) dans le cadre du programme JADE (Cassen, 2012), un pro- jet plus ambitieux est conçu en 2010 avec pour objectifs d'acquérir, traiter, interpréter et restituer des informations spatiales relatives à un des monuments néolithiques les plus réputés du patrimoine architectural européen. Un corpus renouvelé des gravures symboliques s'est ainsi progressive-

${ }^{a}$ CNRS, Laboratoire de recherches archéologiques et architectures (UMR 6566), Université de Nantes, BP 81227, 44312 Nantes. (serge.cassen@univnantes.fr)

${ }^{\mathrm{b}}$ Laboratoire de recherches archéologiques et architectures, Université de Nantes, BP 81227, 44312 Nantes. (valentin.grimaud@univ-nantes.fr)

'Ecole nationale supérieure d'architecture, 6 quai François-Mitterrand, 44262 Nantes. (Laurent.lescop@nantes.archi.fr)

' Master Université de Nantes et Rennes 2. (elvenrobinet@gmail.com)

' Laboratoire d'Archéosciences, Université de Rennes 1, campus de Beaulieu, 35042 Rennes Cedex (nancy.marcoux@univ-rennes1.fr) 
ment constitué au fil des ans après avoir mené, en 2011, un enregistrement topographique et archéologique du cairn et de la tombe à couloir inscrite à l'intérieur. Il s'est agi d'assurer une représentation de l'architecture d'une part, des signes gravés d'autre part, en testant sur ces derniers les modèles interprétatifs proposés depuis une quinzaine d'années, tout en reconstruisant l'image de la première grâce aux avancées récentes des outils numériques à notre disposition (Cassen et al., 2013a et b; Grimaud, 2015).

La dernière dalle $S 12$ formant pavage dans le couloir (fig. 1), dite à juste titre "dalle de seuil " (la numérotation des dalles au sol « $S$ » est distinguée des dalles de plafond " $\mathrm{P}$ ", numérotées depuis l'entrée - aucune nomination n'ayant été proposée par Z. Le Rouzic et E. Shee-Twohig), a retenu l'attention des archéologues à trois reprises au moins dans l'histoire du monument : tout d'abord en 1832 lors de la première exploration connue par M. Cauzique, propriétaire de l'île, qui trouve le bloc soulevé à l'entrée de la chambre, déplacement rapporté à des chercheurs de trésors ayant provoqué l'affaissement partiel de L11 qu'il devait bloquer (Closmadeuc, 1864); puis à l'occasion de la fouille archéologique de G. de Closmadeuc qui entraînera une nouvelle manipulation du bloc pour en sonder les couches sédimentaires sous-jacentes (Closmadeuc, 1884); enfin, lors des travaux modernes de rénovation qui ont abouti à son enlèvement pour assurer un moulage puis son remplacement in situ par un tirage positif (Le Roux, 1981, 1982), opération qui permit la découverte de nouvelles gravures sur la face cachée et d'une lame de hache en fibrolite dans la couche archéologique sous-jacente.

L'état sanitaire de la dalle est aujourd'hui satisfaisant, tenu compte bien entendu des altérations qui ont affecté les angles et les surfaces lors des restaurations sur place (projection de ciment) et lors de son enlèvement, son transport et son stockage (éraflures, écrasements, frottements colorés - bleu, jaune - au contact d'objets modernes). La coloration actuelle ocre à jaunâtre de l'empreinte en élastomère de latex (caoutchouc naturel prévulcanisé et émulsionné dans de l'eau ammoniaquée), gaze textile (tarlatane) et résine faite par J. Airvaux en 1983 (avant fixation sur une coque de plâtre pour les tirages) n'a pas laissé de trace sur l'original, mais a bien sûr arraché de fins éléments de la roche grenue, notamment au creux des tracés, encore attachés sur cette empreinte (fig. 2).

Les gravures détectées dès le Xix ${ }^{e}$ siècle sur la face supérieure de la dalle, usée par les piétinements (cf. les aquarelles produites par W. C. Lukis dans les années 1860, peu précises relativement à S12 - Guernsey Museums \& Galleries), ont pu servir l'interprétation générale de Gavrinis, notamment dans le cadre général des études relatives aux zones de transition et de passage que révèlent en Europe certains

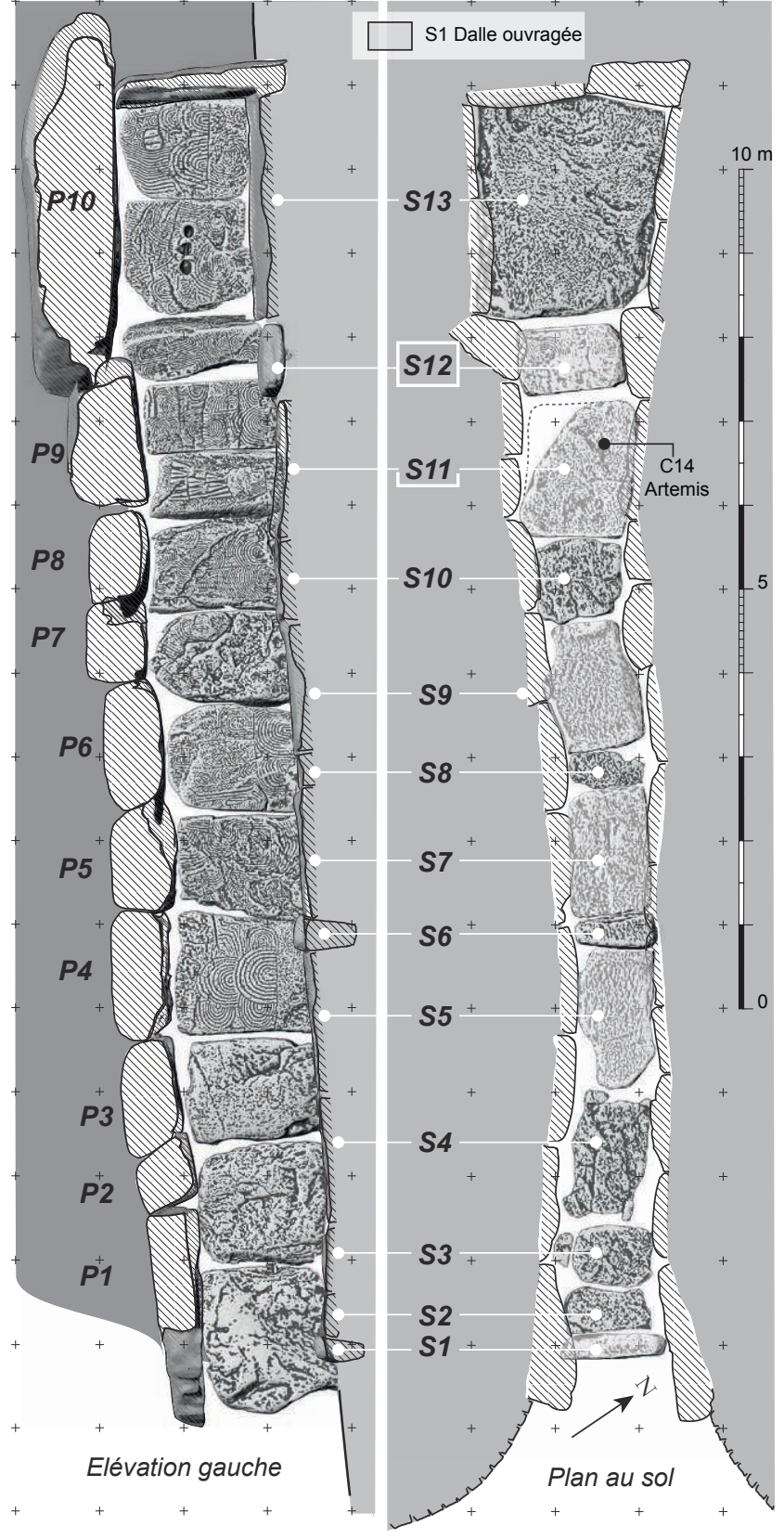

Figure 1 : Plan (sections à mi-hauteur pour les orthostates) et élévation gauche (sud) de la tombe à couloir de Gavrinis (LarmorBaden, Morbihan).

Figure 1: Plan (sections halfway up for orthostates) and left rise (South) of the passage grave of Gavrinis (Larmor-Baden, Morbihan).

éléments architecturaux et signes gravés (Kergal, 1977; Robin, 2009). Les propres levés de G. de Closmadeuc en 1864 sont approximatifs et ne concernent que la tranche 1 (fig. 3). Dans sa livraison de 1873, l'auteur restitue le dessin de la face supérieure ainsi que cette même tranche 1 , mais curieusement en copiant la planche parue dans le 


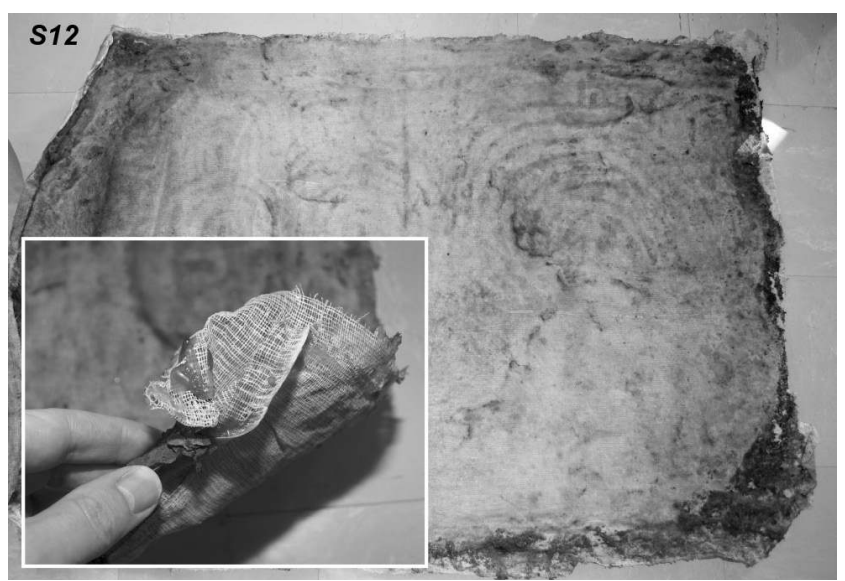

Figure 2 : (Voir planche couleur I) L'empreinte souple de la face supérieure de $S 12$ (élastomère de latex, textile et résine) réalisée en 1983 en vue du tirage en ciment.

Figure 2: (See colour plate I) The negative flexible imprint of the upper side of $S 12$ (elastomer of latex, gauze and resin) prepared in 1983.

Dictionnaire archéologique de la Gaule (DAG 1875) qui est un dessin de l'estampage fait par A. Maître (1885) sur son propre moulage de 1865 conservé au Musée des antiquités nationales (MAN, Douau, 1984). Avec la découverte des nouveaux motifs lors des fouilles de 1884 (Closmadeuc, 1884), les deux tranches sont levées et présentées de concert, mais simplifiées alors que l'on connaît un meilleur dessin inédit de la main de l'archéologue. La plupart des auteurs du $\mathrm{XIX}^{\mathrm{e}}$ et $\mathrm{du} \mathrm{Xx^{e }}$ siècle ne feront d'ailleurs que reproduire le levé dû à A. Maître, souvent sans se soucier de rappeler la source du modèle (Luquet, 1913; Stockis, 1921), un dessin plutôt clair pour l'époque (à partir de ce catalogue, J. Déchelette en 1912, sur le sujet plus large des relations à longue distance, compare déjà le motif en spirale aux exemplaires irlandais). Enfin, St-J.-M. Péquart et Z. Le Rouzic 1927 appuient leur levé de S12 sur la photographie du tirage positif de l'empreinte originale conservée au MAN. Mais il faudra attendre le levé direct sur calque par E. Shee-Twohig pour que l'ensemble des trois faces visibles soit correctement reproduit (Shee-Twohig, 1981).

Après un transfert au dépôt de fouilles de Colpo dans les années quatre-vingts, cette dalle en granite dite de Carnac est aujourd'hui conservée au dépôt archéologique du Morbihan (Vannes) où nous sommes intervenus pour l'enregistrer. Ce travail a pu être mené à bien grâce à l'intervention du Service départemental d'archéologie du Morbihan qui a mobilisé un camion-grue afin que le bloc soit retourné en toute sécurité pour être numérisé et pesé.

L'étude qui suit abordera des questions méthodologiques relatives à l'enregistrement des gravures, évidentes ou altérées, et du support rocheux dans sa morphologie intime pou-
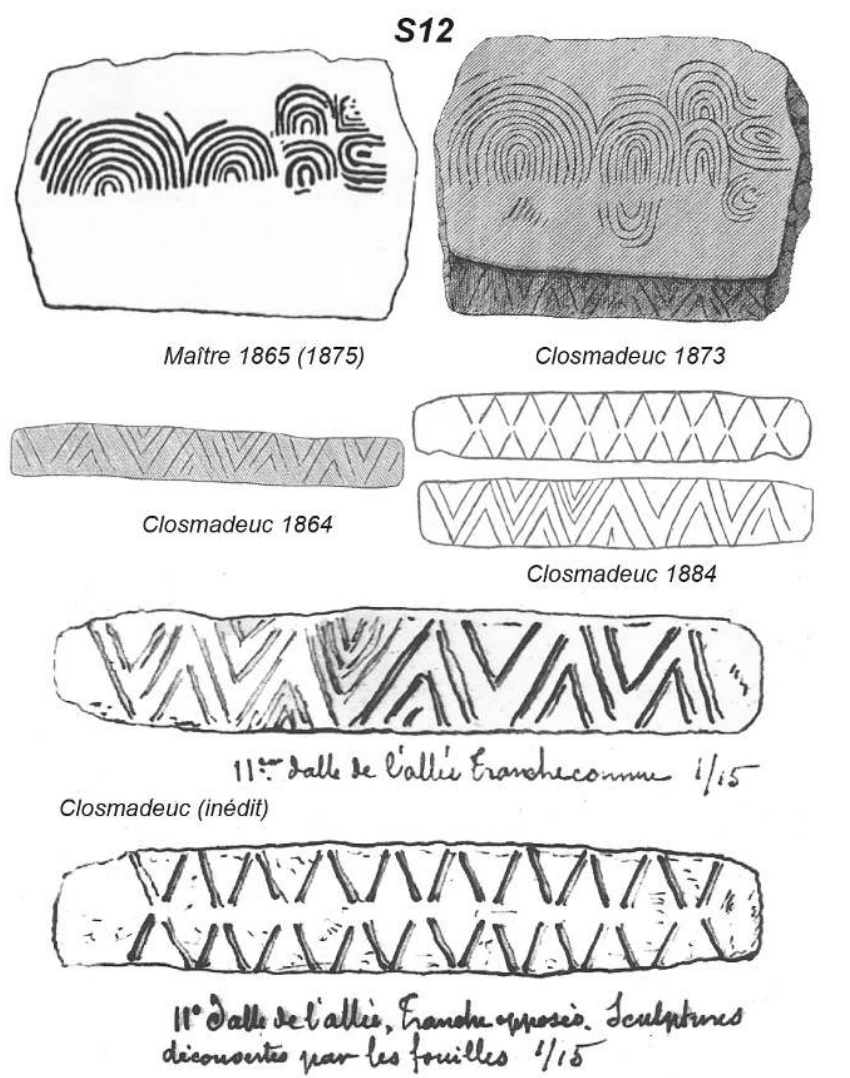

Figure 3 : Levés graphiques sur S12, d'après A. Maître 1875, par estampage sur un moulage de 1865 , et d'après G. de Closmadeuc avant sa fouille $(1864,1873)$ et après son intervention de terrain (1884); le dessin inédit (non daté) est de G. de Closmadeuc (archives LARA, Nantes).

Figure 3: Drawings on S12, after A. Maître 1875, by swindle on a molding of 1865, and according to $G$. de Closmadeuc before his excavation $(1864,1873)$ and after his field work (1884); the drawing by $G$. de Closmadeuc (LARA archives, Nantes).

vant contribuer à la lecture et à l'interprétation des signes. Les différents outils informatiques employés seront testés et permettront un exercice comparatif entre tous les résultats obtenus, notamment en termes de dimensions, volumes et masses. Enfin la conservation inattendue de restes carbonisés dans les creux mâchés des tranches fracturées a conduit à une expérience de datation par le radiocarbone qui participera d'une biographie du monolithe.

\section{L'ACQUISITION ET LE TRAITEMENT DES DONNÉES}

Toutes les faces et les tranches ont pu être numérisées puisque la dalle a été retournée (une séance autorisée par la DRAC de Bretagne). Trois techniques complémentaires 
ont été mises en œuvre pour optimiser cette opération et profiter des possibilités de manipulation de l'objet : la lasergrammétrie, la photogrammétrie et la compilation de clichés sous éclairages tournants. Nous cherchons à comparer les résultats graphiques et topographiques et les « coûts » relatifs (prix des logiciels, temps de réalisation) suivant les outils employés.

\section{Lasergrammétrie}

Pour Gavrinis, le choix d'un enregistrement systématique des surfaces ornées s'est porté, en 2010, sur la lasergrammétrie par triangulation. Le principe consiste, on le sait, à émettre un rayon lumineux dans une direction précise et connue, et d'enregistrer via des caméras la déformation de la projection sur la surface. La relation spatiale entre la source lumineuse et la caméra étant connue, les algorithmes sont capables de déterminer la distance entre la surface et le dispositif de mesure. Il ne reste alors plus qu'à enregistrer en temps réel le déplacement du capteur dans l'espace pour restituer l'objet numérisé. Cette famille de systèmes optiques actifs fournit des résultats précis jusqu'à $1 \mu \mathrm{m}$. Pour numériser la dalle de seuil S12, cette dernière technique sera employée par le biais de l'instrument Krypton K610 (Nikon) en vue d'un enregistrement fin à $0,5 \mathrm{~mm}$ de résolution (prestation effectuée par Y. Lizard du Centre de ressources techniques, Morlaix).

Après nettoyage des données brutes, il en est résulté un nuage de 8674155 points, permettant de détecter les gravures dont la profondeur mesurée sur le nuage de points est de l'ordre du millimètre pour les plus fines. Toutefois, il convient de préciser à ce stade que la granulométrie de la surface de la dalle est très proche de la résolution du relevé, ce qui peut occasionner des erreurs géométriques. Le maillage obtenu se monte à 17335717 triangles dont la résolution correspond à celle du nuage de points $(1 / 2 \mathrm{~mm})$. Ici, le calcul des écarts nous a semblé le meilleur moyen de mettre en valeur les gravures (Lescop et al., 2013; Cassen et al., 2014a). Ces " cartes des écarts" (fig. 4) consistent à visualiser la distance séparant deux maillages. Elles sont très utilisées dans le milieu industriel, en rétroconception, pour contrôler la conformité d'un prototype à son modèle numérique. Le principe consiste à lisser un modèle géométrique haute définition afin de gommer tous les détails de reliefs. Le résultat de l'opération est alors exprimé sous la forme d'un dégradé de couleurs s'étendant du rouge foncé au bleu foncé (fig. 5; voir aussi plus loin la figure 10). Le vert, couleur médiane, correspond à l'endroit où les maillages en haute définition et les maillages lissés s'intersectent. Les valeurs de couleur chaude permettent de montrer les creux car la surface lissée passe dans l'épaisseur de la gravure; les valeurs de couleur froide permettent inversement de signifier les reliefs en saillie (Grimaud, 2015, p. 187). Ce premier objet 3D, rendu sous forme d'un géométral, servira de modèle de référence pour les modélisations par photogrammétrie.

\section{L'enregistrement photogrammétrique}

Le chantier a été mené en deux phases : avant et après le retournement de S12 sur son support en bois. La prise de vue systématique des six surfaces définies du bloc (faces supérieure et inférieure, tranches 1 à 4) a permis d'engager le processus photogrammétrique de restitution des volumes. Au moment où la photogrammétrie se démocratisait, cette formule très économique et rapide méritait d'être testée et comparée aux deux autres approches (lasergrammétrie, compilation de photographies), plus précises mais aussi plus longues d'un point de vue opératoire.

Lappareil photographique utilisé est un Canon EOS 500D avec une focale de $18 \mathrm{~mm}$. Aucun contrôle sur la lumière n’a été opéré, les photos prises en intérieur bénéficiant d'une lumière diffuse. Cependant, certaines faces sont plus exposées que d'autres. Afin d'harmoniser les différences de couleurs, il a été décidé de convertir les photos en noir et blanc. Cette manipulation ne semble pas affecter les reconstructions mais, avec l'expérience acquise, ne serait pas reproduite. Au total, ce sont seulement quatorze clichés pour la face inférieure et vingt-neuf pour la face supérieure qui seront utilisés pour la modélisation de la dalle.

À des fins comparatives, les logiciels de reconstruction 3D à partir de photographies se sont partagés dans un premier temps entre deux programmes en accès libre : 123DCatch v.9b (2012) et Visual SFM (version 0.5.15). À l'heure de la rédaction de cet article et à la faveur de l'évolution des algorithmes, le même corpus photographique est mobilisé afin de comparer les résultats avec les données de 2012 en utilisant 123DCatch v.1.7 (2016) et Agisoft Photoscan v. 1.2.4, ce dernier étant un logiciel sous licence payante. Le développement qui suit montre les quelques manipulations effectuées pour créer un modèle numérique de la dalle, puis les résultats seront comparés au maillage issu du relevé laser inframillimétrique afin de montrer les avantages et les limites des deux techniques. Ce maillage lasergrammétrique est donc considéré comme notre modèle de référence.

\section{Visual SFM}

Les photos de la face inférieure produisent un seul nuage de points ( 450085 points après nettoyage de l'environnement), mais les tranches y sont absentes. La face supérieure est décomposée en deux nuages " non raccords "; la tranche 1 (186033 points après nettoyage) n'a pas pu être assemblée au reste des données $(815777$ points après 

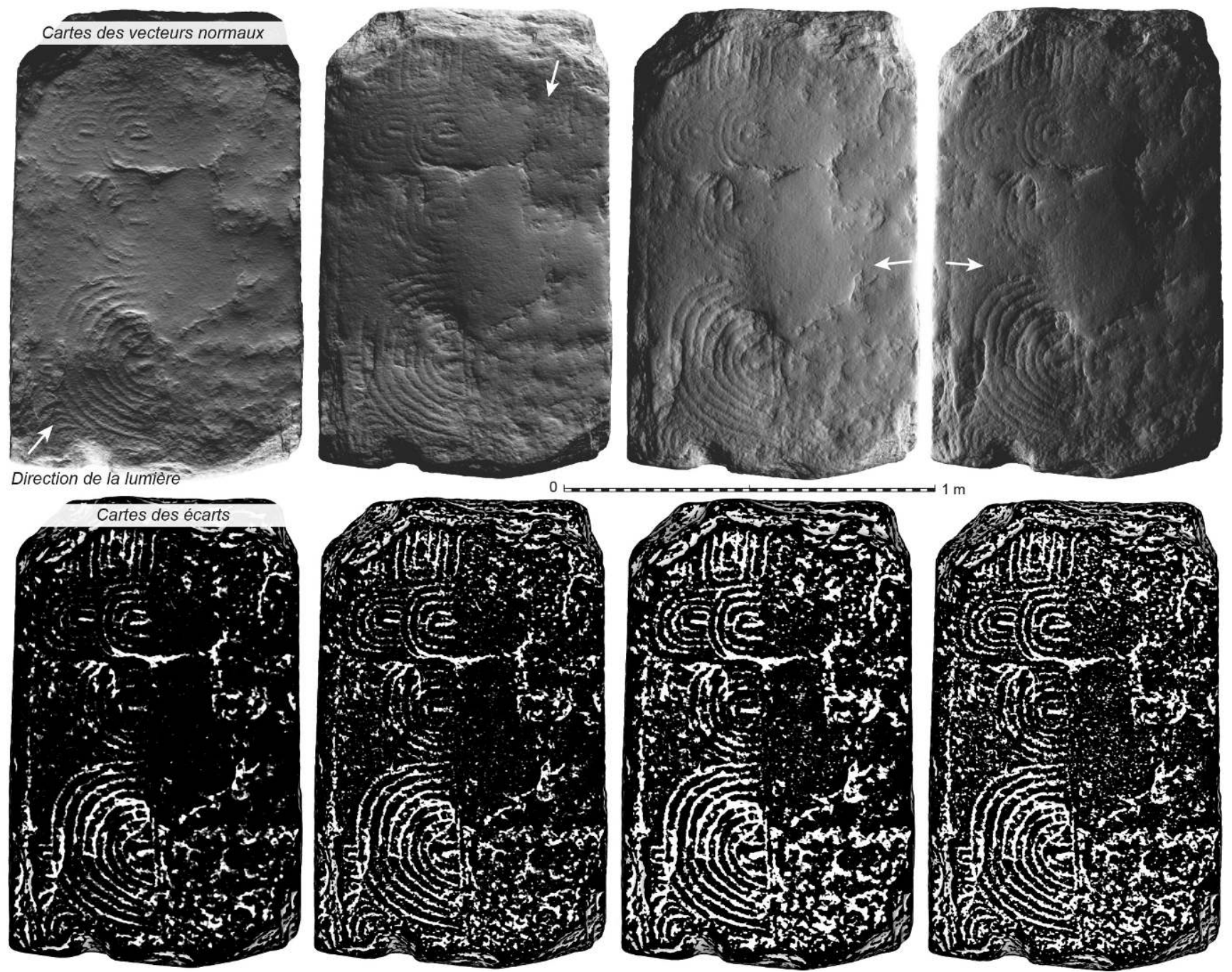

Figure 4 : Deux sources d'information pour détecter et inventorier les gravures sur S12 : en haut, cartes des vecteurs normaux à partir du nuage de points (lasergrammétrie) en variant les incidences «lumineuses »; en bas, cartes des écarts en jouant sur la distance entre les deux maillages haute et basse définitions.

Figure 4: Two information sources to detect and to inventory engravings on S12: at the top, normal vectors map from the points cloud (lasergrammetry) by varying the "bright" incidences; below, deviation map by playing on the distance between both high and low-definition meshings.

nettoyage) car les zones de recouvrement ne sont pas assez importantes. C'est donc avec trois nuages de points qu'il va falloir recomposer la dalle de seuil $S 12$.

Chaque nuage est nettoyé, maillé, mis à l'échelle et repositionné sur le relevé laser de la dalle. Les trois maillages calculés présentent des zones de recouvrement très faibles, voire inexistantes. Il est impossible de reconstituer le modèle avec ces seules données.

Après maillage, la face supérieure est composée de 1600798 triangles, ce qui correspond à une résolution millimétrique; elle est repositionnée sur notre référentiel avec un écart moyen de $0,96 \mathrm{~mm}$. La face inférieure est composée de 881223 triangles, à son tour reposition- née sur le maillage issu du laser avec un écart moyen de $1,51 \mathrm{~mm}$. Le maillage de la tranche 2 est très bruité et lacunaire; il ne s'agit que de lambeaux et il est impossible de le repositionner sur le nuage de points : il est inexploitable pour un géométral archéologique sérieux et pour un rendu minimal des gravures.

Le maillage final est composé de 2564444 triangles, à son tour repositionné sur le maillage de référence avec un écart moyen de $0,98 \mathrm{~mm}$. Il convient toutefois de noter que cet écart moyen s'applique à l'ensemble du volume mais ne décrit pas la résolution des données obtenues avec Visual $S F M$. Avec une densité d'information dix fois moindre, la résolution est de l'ordre d'un point tous les $2 \mathrm{~mm}$. Le mail- 


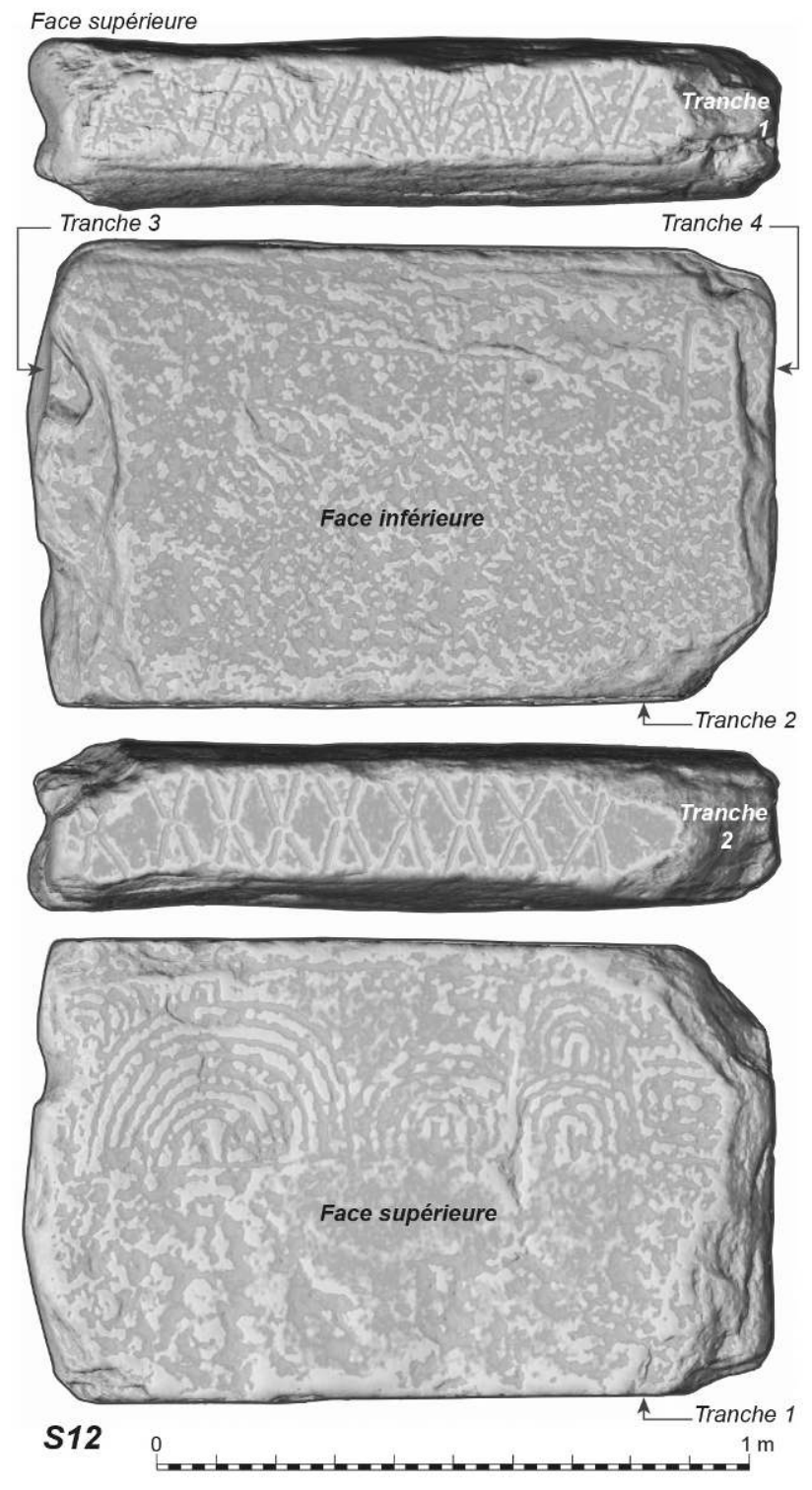

Figure 5 : (Voir planche couleur I) Développé des deux faces et deux chants de S12 d'après une carte des écarts; la visualisation de la distance séparant deux maillages haute et basse définitions permet de faire ressortir les gravures; la gradation des couleurs est explicitée dans le texte.

Figure 5: (See colour plate I) The four faces of S12 developed, according to a deviation map; the visualization of the distance separating high and low-definition meshings allows to highlight engravings; the gradation of colors is clarified in the text.

lage étant ouvert, il n'est donc pas possible de calculer le volume de la dalle sans compléter la géométrie manuellement.

\section{DCatch}

123DCatch est un service gratuit proposé par Autodesk. Destiné au grand public dans le but de promouvoir l'impres- sion 3D, 123D catch fonctionne comme une boîte noire : les photos sont déposées dans l'application et calculées en ligne, puis le résultat est renvoyé : un maillage texturé est proposé en téléchargement, en différents formats, parmi lesquels l'OBJ.

En raison de l'interface proposée par 123DCatch, le chantier est mené en deux étapes. La première consiste à calculer séparément les deux faces de la dalle; la seconde consiste à les assembler. Après calcul et un pré-nettoyage de l'environnement, nous obtenons deux maillages relativement complets et les tranches sont suffisamment recouvertes pour qu'on puisse raccorder les deux faces par la suite.

À l'issue du calcul réalisé en 2012, les faces supérieures et inférieures sont respectivement constituées de 179761 et 121636 triangles, soit 301397 triangles au total. Un premier recalage spatial est réalisé manuellement pour faciliter par la suite le repositionnement automatique (best fit) au sein du logiciel Geomagic Studio 2012. Cette technique va détecter les volumétries similaires pour repositionner au mieux les maillages entre eux. Après fusion dans ce même logiciel d'édition, la surface est composée de 270082 triangles. Ici, la résolution est proche du centimètre.

La même opération est réalisée en 2016 : après suppression de l'environnement, les faces supérieures et inférieures sont respectivement constituées de 562671 et 259371 triangles, soit 822042 triangles au total. Le saut quantitatif est notable. Les maillages sont repositionnés à l'aide de CloudCompare, en transférant les coordonnées des points facilement repérables sur les textures des deux surfaces. La fusion des données a nécessité de supprimer la surface pour ne conserver que les sommets afin de reconstituer un nuage de points, puis d'appliquer l'algorithme de reconstruction de surface Poisson (dans CloudCompare, niveau de subdivision 9). Le maillage ainsi obtenu est composé de 853254 triangles. D'une version à l'autre, la résolution passe pratiquement d'un facteur de 1 à 3 .

Relativement à l'échelle du modèle, un repère centimétrique fut positionné à proximité de la face inférieure. Il a donc servi à mettre cette face aux bonnes dimensions pour l'opération de 2012. La face supérieure a été redimensionnée manuellement pour s'adapter à celle-là. Une part d'erreur non négligeable a pu s'introduire à cette étape. D'ailleurs, lorsque nous repositionnons le résultat $123 \mathrm{DCatch}$ sur le modèle lasergrammétrique, la dalle est trop petite : il faut la mettre à l'échelle selon un facteur de 1,075 , sinon l'écart moyen avec le maillage de référence est de $17,5 \mathrm{~mm}$ (il tombe après mise à l'échelle à $1,78 \mathrm{~mm}$ ). La reconstruction de 2016 s'est directement appuyée sur le modèle de référence issu de la lasergrammétrie. Les résultats issus du même outil, à quatre ans d'intervalle, permettent donc de mesurer l'évolution des algorithmes dans le domaine de la photogrammétrie. 


\section{Agisoft Photoscan}

La reconstruction conduite au sein d'Agisoft Photoscan v. 1.2.4. a été réalisée avec un réglage medium pour l'alignement des photos et la construction du nuage de points dense. De cette manière, la face supérieure est composée de 460000 triangles, et la face inférieure de 230000 triangles (soit un total de 690000). Après repositionnement au sein de Photoscan grâce à des points facilement identifiables avec le maillage de référence, et fusion des points, la surface est composée de 834010 triangles, ce qui correspond à la densité d'information obtenue avec 123DCatch. Il convient toutefois de noter qu'avec un réglage high, la résolution aurait été celle du relevé laser, au prix néanmoins d'un temps de calcul bien plus important.

Une remarque générale doit attirer l'attention sur le fait que la densité du maillage n'est pas la même d'une face à l'autre. Cela provient de ce que l'une des faces contient moins d'irrégularités, si bien que lors de la discrimination du maillage opérée automatiquement par les logiciels de photomodélisation, le nombre de triangles conservé n'est pas équivalent partout, contrairement au laser qui pose les points selon un pas régulier (en fonction de la distance au capteur).

\section{Station photographique sous éclairages tournants}

La technique d'enregistrement des gravures à la tablette graphique est finalisée en laboratoire à partir de photographies numériques frontales faites sur la dalle $S 12$, les gravures étant éclairées par lumière artificielle rasante et tournante (fig. 6). Le centre géométrique est approximativement mesuré à la jonction entre largeur et hauteur de l'objet, l'appareil photographique en station étant manuellement amené à la hauteur de ce repère. Les clichés sont bien entendu déformés par l'objectif choisi (ici 10,5 mm), ce qui ne pose aucun problème puisque le modèle tridimensionnel doit justement permettre de recaler les tracés vectoriels. Reste que cette déformation est bien visible sur les traitements graphiques opérés sur ces clichés compilés.

Le matériel de prise de vue est composé d'un boitier Nikon D5000 (objectif 10,5 mm, f/2.8G ED DX Fisheye-Nikkor) muni d'un déclencheur à distance, les modalités d'acquisition étant constantes (ISO 200; format RAW; ouverture à $\mathrm{f}: 20$ ). Le matériel d'éclairage est limité à une torche à LED (96 led) Kaiser (StarCluster) lumière du jour $\left(5600^{\circ} \mathrm{K}\right.$; 500 lux). Les logiciels payants ou gratuits Adobe Photoshop, PTLens, Hemi/Image Trends autorisent une première correction de la déformation due au grand-angle. Depuis la version CS5 Photoshop, Camera Raw intègre un nouvel outil de correction des principaux défauts des objectifs, autrement

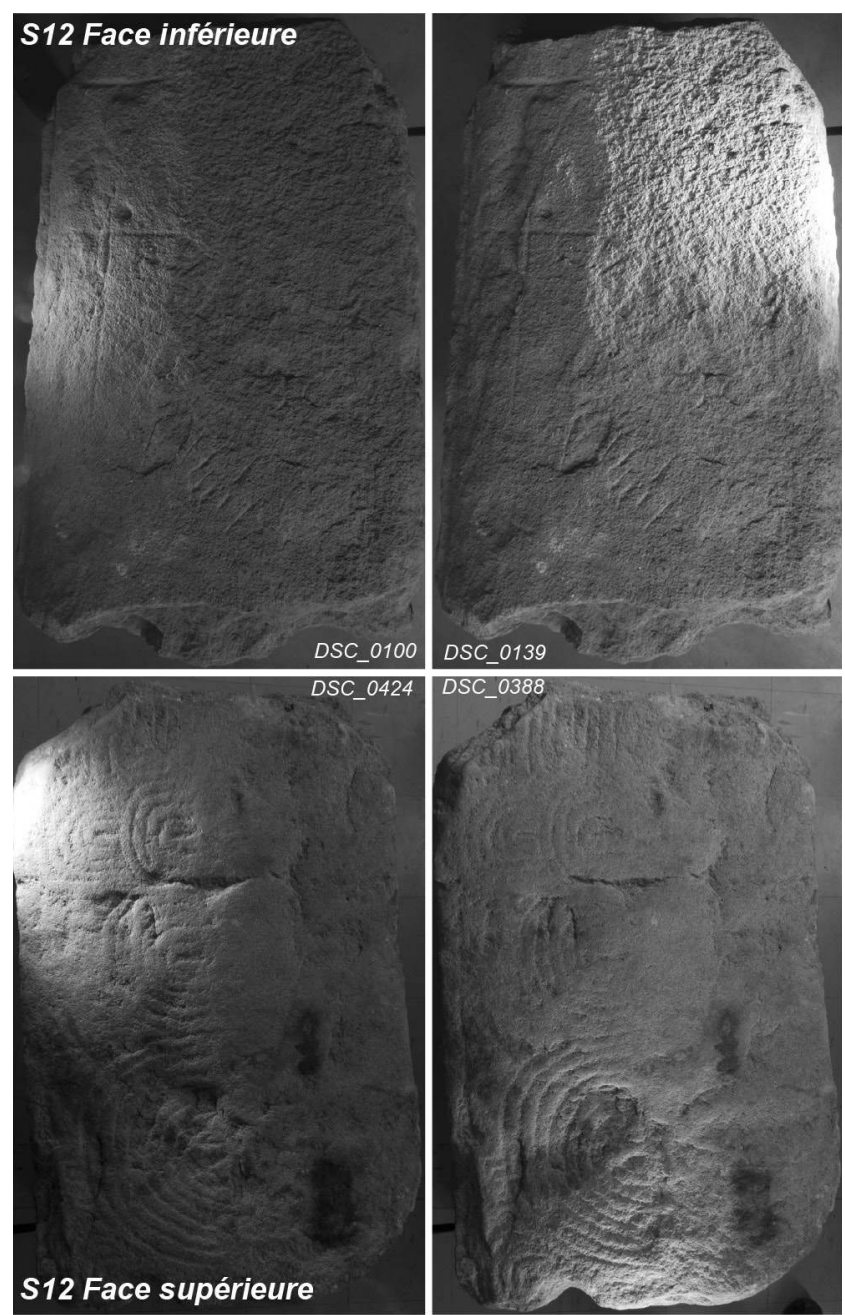

Figure 6 : Détection des tracés et états de surface sur les faces inférieure et supérieure de S12, par compilation de photographies sous éclairages tournants.

Figure 6: Detection of plans and states of surface on the lower and superior faces of S12, by compilation of photos under turning lightings.

dit le vignetage, le chromatisme et surtout les distorsions, l'ensemble étant intégré dans le flux de travail en Raw. Les profils enregistrés dans la base de données couvrent les différents objectifs photographiques du marché.

\section{La face inférieure}

Le corpus photographique couvrant la face inférieure se compose de 80 photos au format NEF $(1,11 \mathrm{Go})$; les 80 photos ont été corrigées au format jpeg pour un traitement plus souple à la palette (utilisation des seules photos DSC_0097 à DSC_0173). Le corpus graphique (1,67 Go) est composé de 28 fichiers vectoriels au format ai et de 3 fichiers de synthèse. Pour localiser les points discutés, nous ferons un choix de secteurs et de tracés dénommés au moyen de lettres et de chiffres. 
Premier constat, vérifiant ce qu'avait noté C.-T. Le Roux en 1982, le martelage d'une bonne moitié de la surface du monolithe a effacé une grande partie de la composition gravée (fig. 7). Ce travail de dressage au percuteur dur et lourd (par analogie avec les résultats expérimentaux par ailleurs obtenus - Vourc'h et al., 2014) est bien entendu d'âge néolithique, et succède à un premier aménagement plus soigné de la surface. Cette seconde intervention, évidemment décalée dans le temps, correspond à l'échelon 1 de notre référentiel en cours de constitution, alors que le premier aménagement serait qualifié à l'échelon 2 ou 3 , autrement dit un dressage poussé et régulier. De profondes griffures parallèles $(\mathrm{F})$ sont par contre le résultat d'une intervention avec un instrument pointu métallique (section en $\mathrm{V}$ ), qui doit être rapproché du soulèvement de la dalle par levier, sans qu'il soit possible de dire s'il s'agit d'une intervention avant 1884 ou après cette première relation de fouille.

Un premier tracé (A1) opère comme limite d'un signe étendu dans le sens de la longueur du monolithe. À peu près parallèle au bord gauche de ce dernier (dans l'orientation donnée en illustration), il s'incurve en haut vers le centre (A2) avant de venir en parallèle au bord supérieur; de même, au bas du motif, le trait est à peu près parallèle au bord inférieur après avoir marqué un angle très arrondi (A3), angle accentué par une dépression dégagée (champlevé) dans la pierre. On note que le tracé supérieur vers le centre de la dalle pointe manifestement vers le haut (A4), mais le palimpseste est par trop effacé pour suivre sans erreur cette limite. Deviné plus que détecté avec assurance, un tracé (A5) qui pourrait être symétrique de A1 apparait sous la surface martelée, mais nous n'avons pu en restituer qu'un seul côté. Enfin un élément plastique doit être souligné en A6, puisqu'il consiste en une arête naturelle dans la roche mais reprise par piquetage et prolongée dans le tracé A2, puis tronquée vers le bas pour rejoindre $\mathrm{A} 1$; cet artifice permet d'adoucir le raccordement du signe supérieur A2/A4 sur A1.
Le tracé $\mathrm{B}$ est normal à $\mathrm{A} 1$, l'angle est parfaitement droit (B1). Il se perd sous la surface effacée. Dans son prolongement vers la gauche, plusieurs clichés (DSC_0112, 0113, 0118, 0129) semblent suggérer son prolongement et même un angle dirigé vers le bas (DSC_0134, 0139). Mais aucun bord opposé à ce trait linéaire n'est apparu sur les clichés sous éclairage symétrique, ce qui, en règle générale, ne permet pas d'accorder du crédit à ces lignes isolées. Plusieurs rectilignes visibles en bordure de la tranche gauche de la dalle sont en réalité des incisions naturelles dans le granite, de part et d'autre d'un miroir de diaclase (cf. plus loin le descriptif des tranches gravées).

Dans le secteur C, juste au-dessus du précédent, plusieurs clichés (DSC_099, 0100, 0106, 0108, 0112, 0113, 0118, 0123, 0124, 0137, 0139, 0157) attestent la présence de deux tracés curvilignes imbriqués $(\mathrm{C} 1, \mathrm{C} 2)$. En partie visibles sur le nuage de points (lasergrammétrie) mais seulement sur leur partie gauche, ils viennent cependant bel et bien recouper A2. Nous avons conservé plusieurs lignes de contrastes en C3 (clichés 0104, 0106, 0112, 0113, 0118), pensant en un nouveau tracé similaire aux deux précédents, mais aucun bord en vis-à-vis n'est finalement apparu, et plusieurs mouvements naturels de la roche semblent pouvoir expliquer ce leurre.

En D, un tracé rectiligne est profondément marqué dans le granite et se perd bien entendu dans la partie droite martelée. Un angle presque droit, mais en réalité aigu à $80^{\circ}$, démontre un prolongement du tracé vers le haut (D2), arrêté par la cassure.

Durant un moment, nous avons également remarqué certains tracés en secteur E, en bas à gauche du monolithe (suivant l'orientation de notre figure). Mais là encore, sans les deux bords symétriques formant la gravure, nous ne pouvons retenir cet indice comme significatif d'un enlèvement de matière.

Figure 7 : (Voir planche couleur II) Synthèse finale en face inférieure de S12 : levé graphique sous éclairages tournants (à gauche) projeté sur le nuage de points maillé (au centre); corrigé de la déformation photographique; dupliqué par rapport à un axe longitudinal (pour comparaison, le levé d'après Le Roux 1982, dessin Y. Lecerf). Inventaire coloré des enlèvements de matière et chronographie des phases de réalisation; les lettres sont appelées dans le texte.

Figure 7: (See colour plate II) Final synthesis on 12 lower face: drawing under rotating lightings planned on the meshed points cloud; correct version of the photographic distortion; duplicated with regard to a longitudinal axis (for comparison, engraving after Le Roux 1982, drawing by $Y$. Lecerf). Colored inventory of the signs and chronography of the phases of realization; letters are called in the text. 


\section{Conclusion intermédiaire, face inférieure}

la face inférieure de la dalle, formant le seuil couloir/ chambre, est un fragment d'un monolithe plus ancien, cassé aux extrémités, probablement dressé dans un premier état. Sa surface est aménagée par dressage régulier avant que ne soit inscrite une composition gravée. Le motif principal suit la configuration de la pierre et semble partagé en deux sous-ensembles, un premier formant le corps principal, un second placé au-dessus mais dans sa continuité. La ligne directrice ainsi dégagée évoque le signe classique dit " en écusson ", ou "idole ", ou " déesse-mère ", d'autant plus que deux signes courbes sur le côté supérieur confortent la dynamique habituelle de ces compositions (" cheveux »). Il s'agit d'un signe phallique selon nos propositions antérieures dont la protubérance visible en bas à gauche pourrait alors s'apparenter aux excroissances latérales mieux connues à l'île Longue ou au Mané Rutual. Le signe supérieur est impossible à restituer, sinon par analogie avec la composition du Mané Rutual (dalle de couverture) où le tranchant d'une lame de hache polie s'oppose au gland du phallus (Cassen et al., 2005).

\section{La face supérieure}

Le corpus photographique couvrant la face supérieure se compose de 70 photos au format NEF (742 Mo); 68 photos ont été corrigées au format .tif, et 61 au format .jpeg pour un traitement plus souple à la palette (utilisation des photos DSC_0365 à DSC_0425). Le corpus graphique (1,98 Go) est composé de 48 fichiers vectoriels au format ai et de 4 fichiers de synthèse. Ici encore, pour localiser les secteurs discutés, nous nommerons les tracés au moyen de lettres et de chiffres.

Le poli d'usure est manifeste sur l'ensemble de la surface, mais il n'est pas plus développé que sur les autres dalles de sol du couloir; les gravures partiellement effacées sur S12 sont simplement mieux à même de révéler cette abrasion marquée. Nous y reviendrons en superposant les courbes de niveaux. Sur le secteur inférieur (en suivant l'orientation arbitraire donnée dans la fig. 8), la surface est aussi affectée d'un martelage assez grossier (état de surface 1) qui a en partie oblitéré les gravures.

Deux tracés plus ou moins rectilignes et parallèles s'opposent au reste de la composition, fondamentalement curviligne (arcs radiés), et limitent un panneau qui semble cantonné à une moitié de la dalle dans son état actuel. Le tracé $\mathrm{L}$ est à peu près parallèle au bord naturel de la dalle (éponte dans le granite ou miroir de diaclase) et semble faire écho à une ligne semblablement orientée mais naturellement inscrite le long du bord opposé sur la face inférieure. Les segments F1, F2 et F3 sont dans le prolongement l'un de

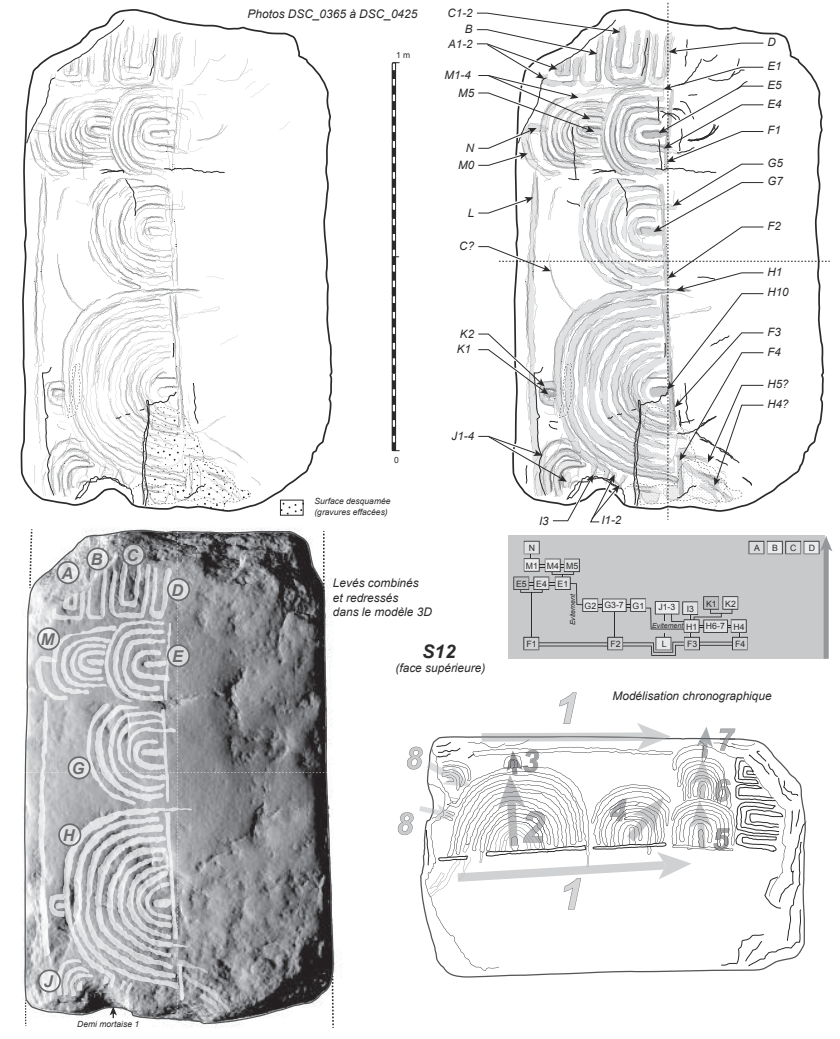

Figure 8 : (Voir planche couleur III) Synthèse finale en face supérieure de S12. Inventaire des contours des signes levés sous éclairages tournants (en haut à gauche), inventaire coloré des enlèvements de matière et chronographie des phases de réalisation (en haut à droite); les lettres sont appelées dans le texte. Synthèse des différents motifs, de $\mathrm{A}$ à $\mathrm{M}$ (en bas à gauche). Modélisation des grandes phases de réalisation et dynamique interne (en bas à droite).

Figure 8: (See colour plate III) Final synthesis on the superior face of S12. Inventory of the outlines of the signs raised under rotating lightings, colored inventory of the signs and chronography of the phases of realization; letters are called in the text. Modelling of the main phases of realization and internal dynamics.

l'autre mais ne se touchent pas et forment une ligne légèrement incurvée, même sur le modèle redressé. En fait, les trois segments « supportent » chacun un motif d'arcs radiés.

Ces trois motifs d'arcs radiés sont donc juxtaposés le long d'une rectiligne.

- Le plus grand $(\mathrm{H})$ est composé de 9 arcs débutant sur un petit segment droit (H10) et s'achève sur un autre petit segment (K1) en conclusion des arcs concentriques. On note un prolongement inexpliqué du dernier arc extérieur en $\mathrm{H} 1$. Une incertitude persiste sur plusieurs témoignages de tracés en $\mathrm{H} 4$ qui pourraient former une prolongation sinueuse du motif précédent. Mais la reprise de cette surface travaillée au cours de la taille du bloc a pu introduire des sortes de « linéations » dues au travail au percuteur. 
- Le motif intermédiaire (G) est fait de 6 arcs radiés et d'un segment central (G7). Ici encore, un arc outrepasse la base rectiligne et se prolonge sur 2 à $3 \mathrm{~cm}$ (G5). Enfin, en $\mathrm{C}$, demeure une interrogation sur une courbe donnée par certains clichés mais non validée par la détection du côté opposé du creux; elle a, pour assurer sa crédibilité, d'être à peu près concentrique aux autres, mais faute d'éléments intermédiaires, il est difficile de retenir son tracé.

- En E, quatre arcs radiés partent d'un segment droit (E5). Et encore une fois, un arc se prolonge au-delà de sa base rectiligne (E4).

- Superposé au motif E, un quatrième motif (M) se compose également de quatre arcs radiés, à leur tour surmontés d'un segment droit $(\mathrm{N})$ et d'un cinquième arc $(\mathrm{M} 0)$ plus englobant mais dont il manque une moitié disparue à l'emplacement de la cassure du monolithe.

- Dernier assemblage de signes dans ce secteur du monolithe, la composition A-B-C-D comporte des tracés symétriquement disposés : $\mathrm{D}$ est un signe isolé qui trouve un correspondant en $\mathrm{B}$, tandis que $\mathrm{C} 1-2$ est un double signe concentrique dessiné sur le modèle de $\mathrm{D}$ mais qui trouve son écho véritable en A1-2. La juxtaposition de ces quatre ensembles forme une sorte de limite rectiligne tangente au motif E.

À l'autre extrémité de la dalle, trois signes courbes concentriques $(\mathrm{J})$ viennent à toucher le motif $\mathrm{H}$. Ils sont interrompus par la cassure et sont difficilement détectables en photographies sous éclairage rasant.

\section{Conclusion intermédiaire, face supérieure}

Malgré une usure considérable de la dalle sous l'action des piétinements des visiteurs pendant plus d'un siècle, il semble bien que sa surface n'ait été gravée que sur une moitié. Quatre motifs d'arcs radiés se juxtaposent et se superposent pour remplir cet espace limité par des tracés rectilignes. À une extrémité de la composition, des signes emboîtés plus quadrangulaires changent son rythme et sa géométrie. Mais des cassures ont amputé le développé de ces gravures, preuve d'un état plus important que celui observé aujourd'hui; la même observation se répète sur un dernier motif de signes concentriques à l'autre extrémité du monolithe.

\section{Les tranches 1 et 2}

Les gravures y sont bien préservées, et le travail sur les éclairages virtuels autorise par conséquent des rendus peu ambigus (fig. 9), il n'est donc pas apparu utile d'établir de stations par images compilées. Les contours restitués sont directement issus des modèles 3D.

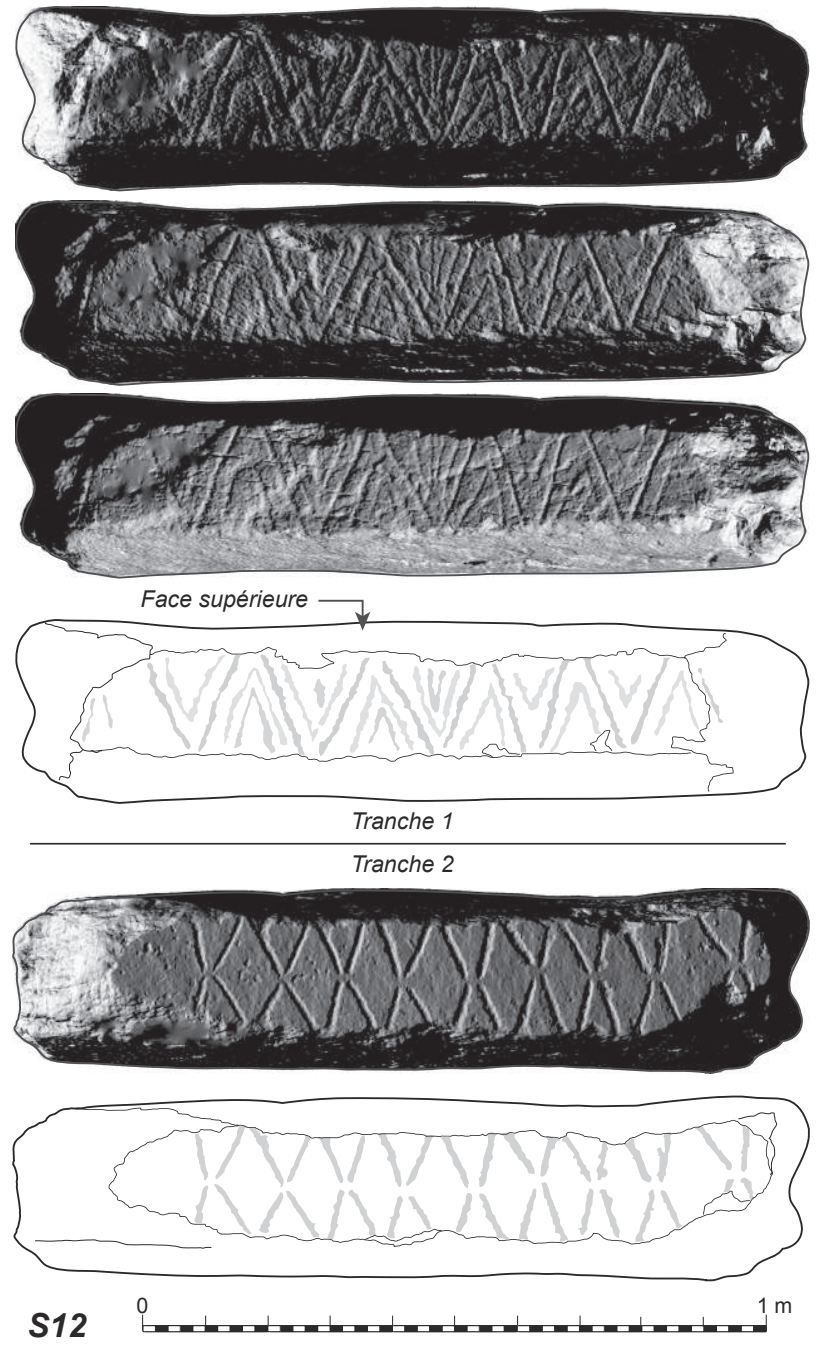

Figure 9 : Détection des tracés sur les tranches 1 et 2 de $S 12$, à partir des cartes de normales. Transfert graphique des gravures. Figure 9: Detection of engraved lines on edges 1 and 2 of S12, from the normal maps. Graphic transfer of the engravings.

\section{UNE DISCUSSION SUR LES RÉSULTATS COMPARÉS}

Les modèles calculés selon les différentes solutions logicielles seront confrontés entre eux, et l'un d'entre eux (123DCatch) sera même l'objet d'un comparatif de performances entre la version utilisée en 2012 et son actualisation opérante en 2016. Au-delà de la morphologie des supports, les gravures recensées dans le modèle tridimensionnel seront ensuite superposées au levé de terrain effectué par éclairages tournants. 


\section{La reconstruction $3 \mathrm{D}$}

Les deux maillages issus de la photogrammétrie sont comparés au modèle lasergrammétrique qui est considéré comme une source "juste ", la technique ayant été éprouvée à maintes reprises. Les deux maillages sont repositionnés grâce à une opération automatique (best fit). De cette manière, les différents maillages partageront la même orientation dans la boîte englobante et les dimensions pourront être comparées. La qualité de ces maillages issus de la photogrammétrie sera donc appréciée par confrontation au maillage de référence. Et l'analyse sera possible grâce aux cartes des écarts. Le tableau 1 résume les différentes dimensions issues des deux opérations de relevé.

La comparaison des maillages issus de la lasergrammétrie et d'123DCatch est illustrée par la figure 10 (en jaune : le maillage 123DCatch est au-dessus du maillage lasergrammétrique). Nous parlons ici de l'assemblage des deux faces de la dalle, assemblage réalisé à partir des tranches produites sous 123DCatch. L'écart représente la part d'erreur produite par le repositionnement automatique (best fit). Il y a bien des gravures détectées, mais elles sont faiblement prononcées, et donc peu d'éléments sur lesquels les algorithmes puissent s'attacher. De plus, nous constatons que les bords du mail- lage 123 Catch ont tendance à se voiler et à s'éloigner de la réalité. Cependant, nous pouvons estimer que l'écart moyen de $1,78 \mathrm{~mm}$ est tout à fait acceptable.

De tels résultats n'apparaissent pas dans le travail effectué avec VSFM. Les maillages ont d'abord été positionnés sur le maillage de référence avant d'être fusionnés. Il est donc normal que l'écart moyen soit ici plus faible que le précédent. En ce qui concerne la face inférieure, une grande étendue bleue est présente sur la partie droite, et plutôt en partie basse (dans l'orientation arbitraire donnée par la figure 10). Cela signifie que cette partie du maillage VSFM se situe derrière le maillage de référence (entre 2 et $4,5 \mathrm{~mm}$ ). Cette zone correspond à peu près à la partie piquetée. La surface étant moins régulière, les hauteurs semblent avoir été exagérées lors de la production du nuage de points. Une couverture photographique plus dense aurait peut-être pu éviter ce problème.

Les cartes d'écarts servant à la comparaison du maillage de référence et des maillages 123 DCatch commencent à révéler des gravures, notamment les plus évidentes sur la face supérieure (arcs radiés) et sur les tranches (motifs losangés). Cela montre que le maillage 123DCatch n'est pas assez dense pour suivre les microreliefs générés par les autres gravures. Sur les cartes des écarts de VSFM, les gravures sont indécelables du fait des résolutions évoquées plus haut (fig. 11).

\begin{tabular}{|l|c|c|c|c|c|}
\hline Technique & Longueur $(\mathrm{mm})$ & Largeur $(\mathrm{mm})$ & Epaisseur $(\mathrm{mm})$ & Volume $(\mathrm{m} 3)$ & Ecart moyen $(\mathrm{mm})$ \\
\hline Lasergrammétrie & 1274 & 794 & 296 & 0,218 & - \\
\hline 123DCatch (2012) & 1272 & 797 & 298 & 0,225 & 1,78 \\
\hline 123DCatch (2016) & 1279 & 856 & 310 & 0,215 & 1,19 \\
\hline VSFM & 1279 & 814 & 296 & - & 0,98 \\
\hline Photoscan & 1295 & 868 & 313 & 0,222 & 1,38 \\
\hline
\end{tabular}

Tableau 1 : Mesures du monolithe prises sur le modèle tridimensionnel, et calculs des volumes selon les programmes utilisés. Table 1: Measures of the monolith extracted from the three-dimensional model, and calculations of the volumes according to the different programs.

Figure 10 : (Voir planche couleur IV) Comparaison entre les maillages issus de la lasergrammétrie et de la reconstruction photogrammétrique : à gauche avec 123DCatch (Autodesk) avec un écart moyen $=1,78 \mathrm{~mm}$; à droite avec Visual SFM avec un écart moyen $=0,98 \mathrm{~mm}$ (écarts peu significatifs puisque les maillages 123DCatch et Visual SFM sont assemblés grâce au maillage issu de la lasergrammétrie). L'échelle colorée des profondeurs est graduée en $\mathrm{mm}$.

Figure 10: (See colour plate IV) Comparison between meshings from lasergrammetry and from photogrammetric reconstruction: on the left 123DCatch (Autodesk) with an average gap = 1,78 $\mathrm{mm}$; on the right Visual SFM with an average gap $=0,98 \mathrm{~mm}$ (little significant gaps because 123DCatch and Visual SFM meshings are assembled thanks to the meshing from the lasergrammetry). The colored scale of the depths is increased in $\mathrm{mm}$.

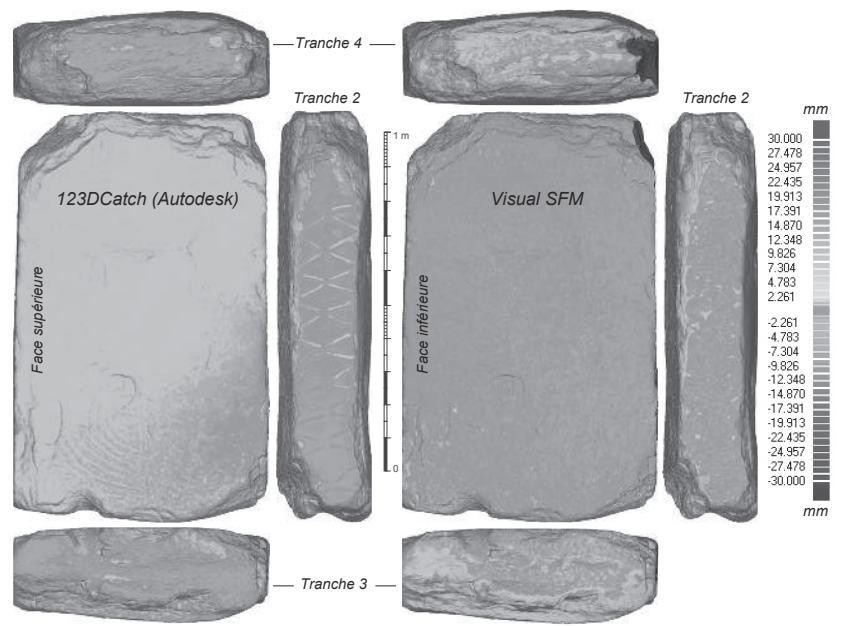




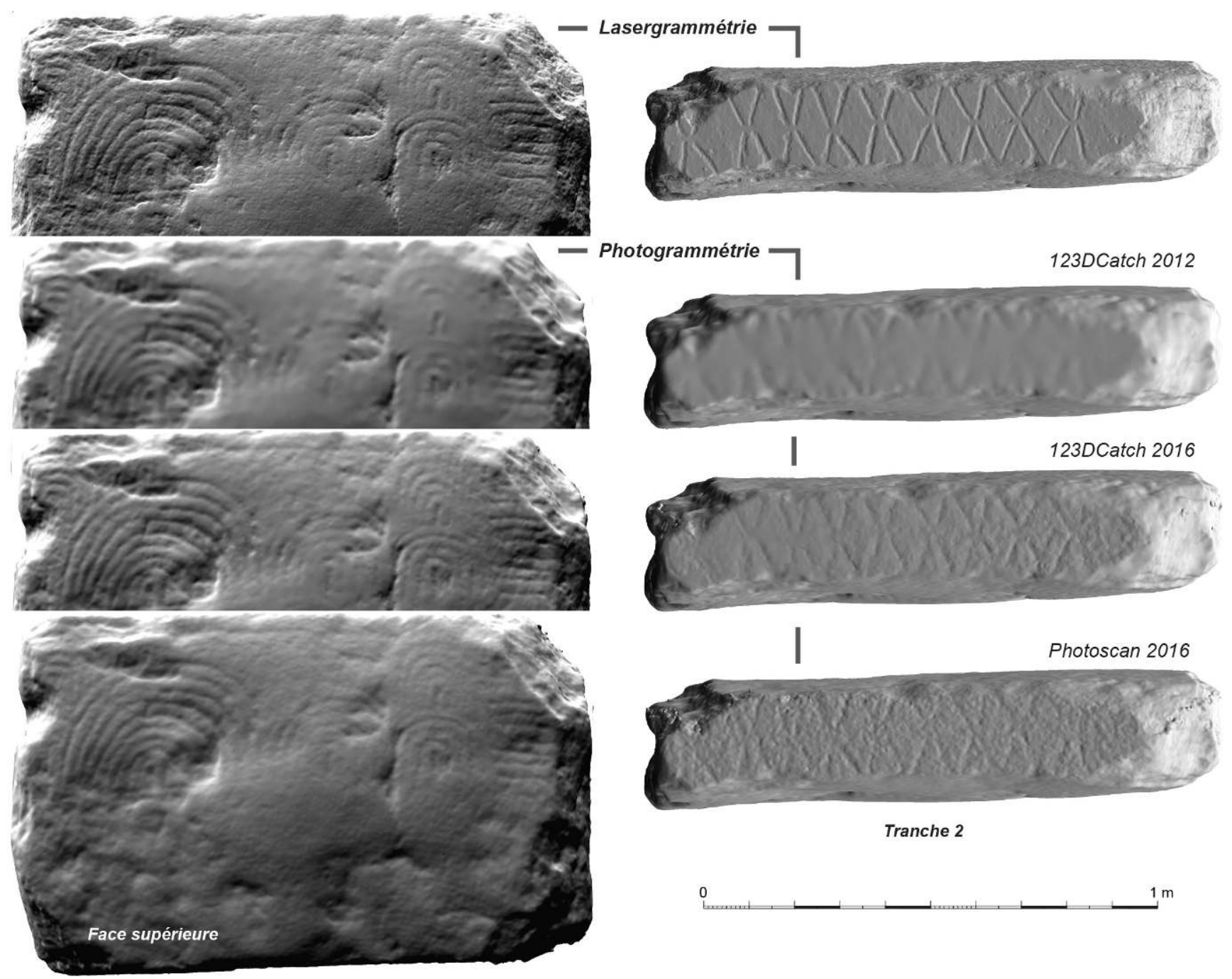

Figure 11 : Comparaisons de deux des surfaces de $S 12$ (face supérieure et tranche 2) selon les rendus respectifs de trois programmes de traitement photogrammétrique, en accès libre (123DCatch) ou payant (Photoscan), le modèle lasergrammétrique étant pris en référence. Figure 11: Comparisons of two surfaces on S12 (upper side and section 2) according to the respective depictions by three photogrammetric programs, the lasergrammetric model being in reference.

Enfin, les données lacunaires sont visibles sur les cartes des écarts. Pour le résultat d'123DCatch, nous le voyons particulièrement bien sur la tranche située sous la face supérieure (en jaune). Pour le résultat VSFM, nous le constatons sur la tranche située sous la face inférieure, sur toute la longueur de l'arête supérieure, ainsi que sur la tranche à droite de la face supérieure, là où le passage du bleu au jaune est rapide.

Au-delà du fait que l'objet en 3D soit aisément manipulable dans toutes les directions, ce qui est un pas considérable par rapport aux techniques classiques d'enregistrement et de présentation antérieures, le résultat surfacique nous paraît très satisfaisant (fig. 10). Si les premiers enregistrements photogrammétriques restaient globalement flous par rapport au modèle issu de la lasergrammétrie, la manipulation du même corpus de clichés avec des outils plus mûrs montre bien la possibilité de mieux qualifier les états de surface - sans toutefois égaler la précision du modèle obtenu en lasergrammétrie. Le rendu même de la granulosité du granite permet de différencier les grands états de surface, entre le poli de la face supérieure et le piqueté fin ou le martelage de la face inférieure. Enfin, les gravures apparaissent sur toutes les faces, sans prétendre pourtant à la précision et à la résolution des deux méthodes précédentes (lasergrammétrie, images compilées). L'ensemble des données acquises aujourd'hui en vue d'une reconstruction photogrammétrique constituera donc, dans quelques années, des corpus précieux pour de nouvelles études sur ces objets; 
corpus qu'il convient dès aujourd'hui d'archiver pour les générations futures.

En traçant maintenant les courbes de niveaux à l'équidistance de 5 et $10 \mathrm{~mm}$ sur la face supérieure de S12 (fig. 12), il est particulièrement intéressant de corréler ces courbes avec la disparition totale ou partielle des gravures, prouvant, s'il en était besoin, que l'usure due aux piétinements avait immanquablement effacé ces tracés en partant des points hauts de la surface de la pierre. Dans le cadre d'un bilan sanitaire, une cartographie plus précise encore permettrait de localiser l'emprise exacte de ce poli d'usure, tout en donnant une indication précieuse à propos du processus d'altération dans le granite de Carnac.

\section{L'inventaire des signes gravés}

Le travail infographique mis en œuvre à Gavrinis pour établir un premier catalogue brut des gravures (objectif du rapport d'opération programmée 2012) s'est fondé sur un fonds d'images complémentaires (fig. 13). À partir du nuage de points de la lasergrammétrie, deux ensembles de documents sont créés : l'un propose un certain nombre d'éclairages virtuels rasants autour de la dalle, l'autre suggère plusieurs cartes des écarts à différentes "profondeurs" de plans de section (4 cartes sélectionnées en fig. 4). Depuis ces

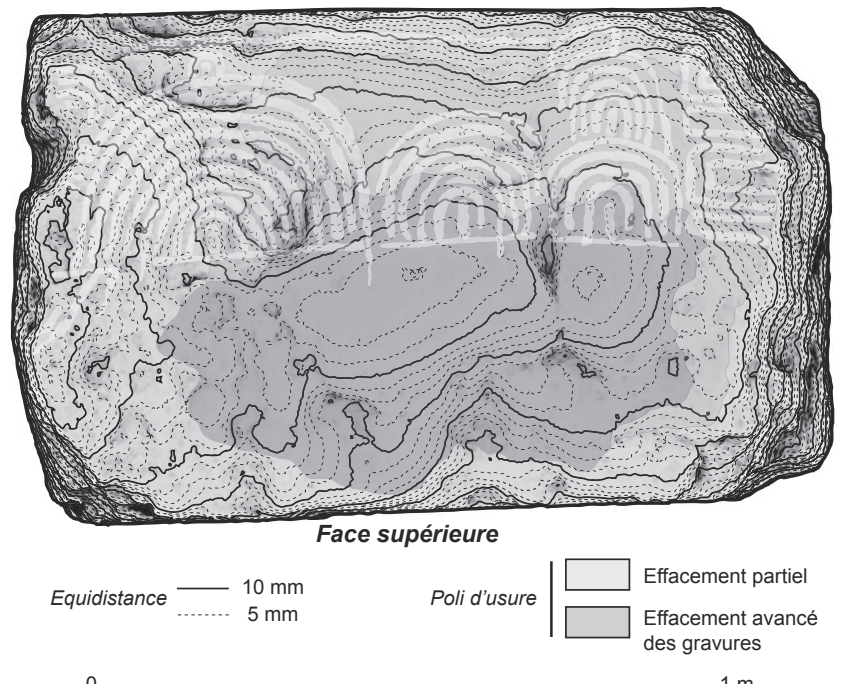
$1 \mathrm{~m}$

Figure 12 : (Voir planche couleur IV) Microtopographie de la face supérieure (modèle numérique de surface) montrant la relation entre le poli d'usure dû au piétinement des visiteurs et les parties hautes du relief de la dalle.

Figure 12: (See colour plate IV) Microtopography of the monolith upper side (digital model of surface) showing the relation between the wear polished due to the passage of the visitors and the high parts of the relief. dernières, une vectorisation des zones contrastées permet de visualiser les contours des creux afin d'informer l'opérateur qui intervient à la tablette graphique pour dessiner les tracés. Ce travail s'effectue, en règle générale, à partir d'une de ces cartes des écarts, mais le graphiste a tout loisir, à partir des différents calques superposés dans le fichier source, de consulter ou bien une carte des écarts supplémentaire, ou encore une image en "carte des normales" (normal map) qui joue sur des sortes d'éclairages virtuels.

Nous avons ici fait le choix de produire ce catalogue en sollicitant une dessinatrice (ER) non informée du corpus et non spécialiste du Néolithique et de l'art rupestre Holocène. La chronographie des gravures n'est ici pas recherchée, étant donné les outils employés, mais elle est cependant partiellement possible. Le résultat obtenu est donc proche d'un travail "en aveugle ", et c'est ce levé qui sera comparé à la synthèse donnée par la méthode des photographies compilées.

Le levé des deux faces du monolithe, opéré à partir de l'acquisition lasergrammétrique, est présenté en figure 14 . Il semble à première vue peu éloigné de notre précédent exercice se fondant sur les éclairages tournants et, à cet égard, la précision de $0,5 \mathrm{~mm}$ du levé numérique au scanner 3D paraît suffisante; il s'est avéré en réalité que cela correspondait à la granulométrie du support, ce qui a par

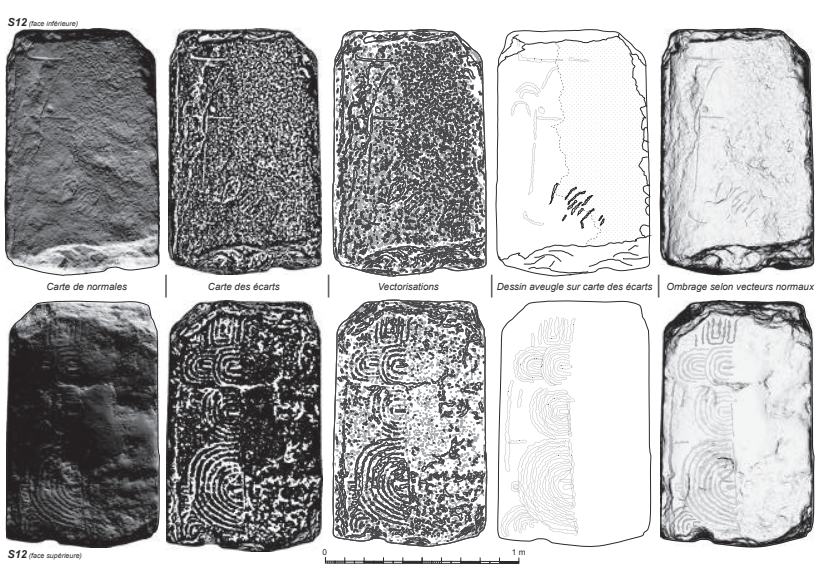

Figure 13 : (Voir planche couleur V) Sources et traitements de l'information numérique pour détecter les gravures sur les faces supérieure et inférieure de $S 12$ et les représenter en plan. De gauche à droite : cartes de normales depuis le nuage de points, cartes des écarts, vectorisation de la carte des écarts, extraction des signes par un opérateur non informé, intégration des signes dans la face traitée en ombrage selon les vecteurs normaux.

Figure 13: (See colour plate V) Sources and processings of digital information to detect engravings on the superior and lower faces of S12 and to represent them in plan. From left to right: normal maps from the points cloud, deviation maps, vectorisation of the deviation map, extraction of the signs by uninformed operator, integration of the signs in the face treated in shader according to the normal vectors. 
ailleurs posé des problèmes pour le traitement numérique de l'information. Dans le détail (fig. 14), plusieurs nuances d'importance permettent de souligner les erreurs d'appréciation sur les enlèvements de matière, par l'une ou l'autre méthode, quand bien même le tracé aurait été parfaitement détecté dans les deux cas; les complémentarités graphiques obtenues en jouant sur ces méthodes apparaissent comme un résultat tout aussi important. L'intérêt de ce comparatif est bien sûr de rapprocher deux levés réalisés de façon tout à fait indépendante.

\section{Face inférieure}

La dynamique d'ensemble de la composition est bien partagée entre les deux méthodes, ce qui n’a rien de surprenant, et valide la reconnaissance d'un motif, dit phallique, connu par ailleurs en Morbihan (et désormais sur une stèle néolithique en Bourgogne, anatomiquement connecté à un anthropomorphe - Cassen et al., à paraître).

- Des différences d'appréciation apparaissent cependant sur le signe supérieur tronqué (dans l'orientation arbitraire donnée par notre dessin), essentiellement au niveau de son coin gauche qui marque un angle plus aigu que droit sur le

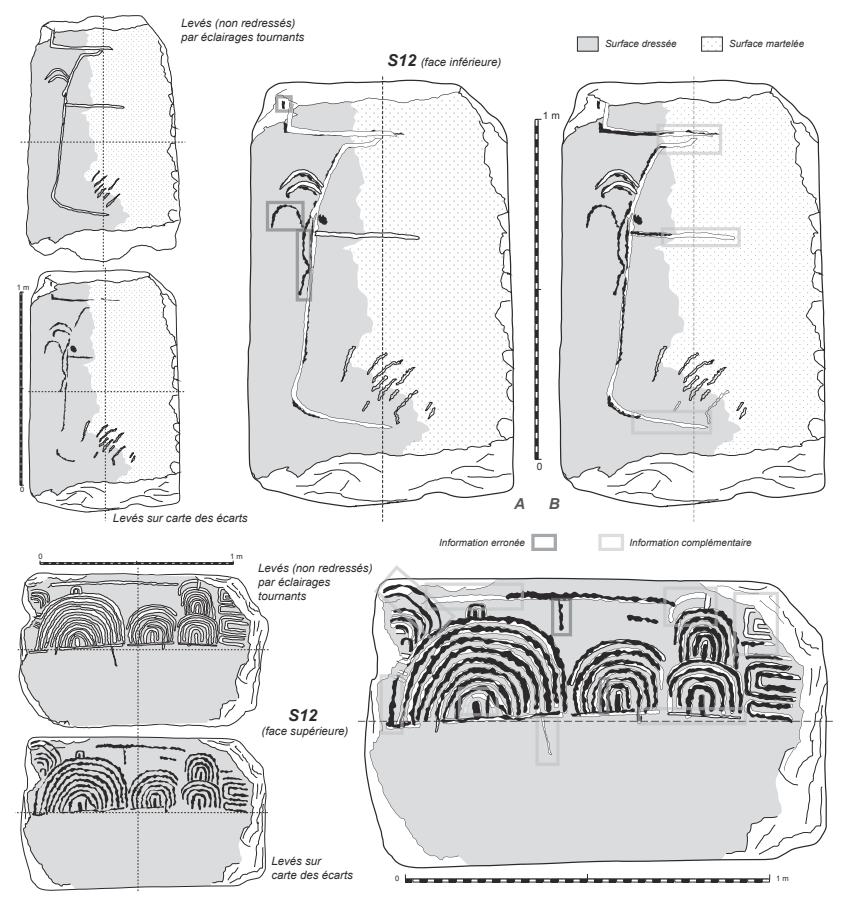

Figure 14 : (Voir planche couleur VI) Comparaison signe à signe par compilation des informations extraites des cartes des écarts et du levé par éclairages tournants sur les faces inférieure et supérieure de S12.

Figure 14: (See colour plate VI) Comparison sign to sign by compilation of the information extracted from the deviation maps and from the turning light process, on the lower and superior faces of S12. levé photographique. Notre choix se portera sur cette dernière proposition car les occurrences y sont répétées alors que le segment suggéré à travers la carte des écarts est unique.

- Une erreur manifeste dans le travail sur la carte des écarts se note sur le flanc gauche du motif " phallique " (fig. 14, A), confondu avec une arête naturelle en ressaut. On sait que cette arête est reprise par piquetage pour former la base du "gland ", ce qui explique cette erreur. De même, l'excroissance courbe qui s'appuie sur ce renflement n'a pas été retenue dans le levé photographique car il s'agit d'un repli naturel dans la roche, seulement concordant avec les vrais signes recourbés gravés juste au-dessus. Leurre visuel et contamination visuelle ont sans doute ensemble provoqué ce choix de l'opérateur travaillant à la tablette graphique.

\section{Face supérieure}

La concordance des tracés est ici aussi respectée, sauf quand le redressement des dessins sur les photographies déformées par l'objectif atteint ses limites, d'une part, et quand le contour du tracé en creux donné par la carte des écarts englobe tous les détours parasites générés par cette dernière, d'autre part.

Inutile d'insister sur les complémentarités de tracés soulignés sur la figure 14. Certains signes rectilignes très effacés n'apparaissent pas sur le levé au scanner à $0,5 \mathrm{~mm}$ de résolution alors qu'ils sont apparents sur la photographie. En revanche, sur la partie inférieure du monolithe, plusieurs tracés inédits révélés par le scanner ne sont pas détectés par la photographie, au rebours de la situation habituellement rencontrée jusqu'ici. Notre explication aimerait mettre en avant la configuration des surfaces enregistrées, très tourmentées au niveau de la cassure et qui, pour cela, ne peuvent pas bien se prêter à des éclairages incidents étant donné le nombre des microreliefs masquant le creux des gravures, $a$ fortiori si elles sont altérées.

\section{Tranche 1}

Les deux côtés de la dalle S12 sont des diaclases naturelles du granite liées aux phénomènes tectoniques (fig. 9). Aucune préparation ne les a affectées, le miroir de faille étant parfaitement plan. C'est d'ailleurs pour cette raison que le seul enregistrement lasergrammétrique sera ici utilisé et commenté, la lecture des gravures n'opposant pas de difficulté majeure.

La tranche 1 est néanmoins plus abîmée en surface que son opposée, probablement du fait de manutentions (bardages) néolithiques car les éraflures et écrasements sont anciens. Des altérations plus fortes marquent les extrémités de la composition, au niveau des fractures.

Cette composition consiste en signes rectilignes gravés, dits en chevrons, sur toute l'étendue de l'éponte originelle 
dont il manque probablement une partie des deux arêtes parallèles formant l'angle de raccord avec les faces inférieure et supérieure. Malgré cette incertitude, il n'est pas évident que toutes les pointes supérieures des triangles aient été en contact (tracés bleus, fig. 9). On note un changement de rythme entre deux secteurs, deux ou trois triangles emboîtés faisant la différence.

\section{Tranche 2}

Si la surface est ici mieux conservée, les arêtes sont plus endommagées. Les signes rectilignes forment des sortes de triangles opposés par le sommet, déterminant des losanges, à moins qu'il ne s'agisse de « dents de loups » de part et d'autre d'un axe imaginaire. On note que les segments sont moins obliques aux deux extrémités de la composition, comme s'ils la limitaient, et d'ailleurs, l'espace est libre à gauche des derniers traits gravés.

\section{Finalement, en comparant au levé antérieur}

Le travail mené par E. Shee-Twohig (1981) est, de loin, le meilleur levé que l'on puisse comparer à nos deux ensembles de résultats. Le premier constat, découlant de la superposition des rendus graphiques respectifs, celui de notre collègue irlandaise et celui dû aux images compilées (fig. 15), montre bien le problème de distorsion provoqué par des levés directement effectués sur les supports (estampages, frottis, transferts au crayon sur feuille translucide ou transparente); la projection (vue planaire) est généralement erronée. Mais l'auteur semble avoir ici dissocié le levé du support de celui des signes proprement dit, ces derniers étant globalement respectés à travers le processus projectif, en raison d'un traitement motif par motif, en plusieurs feuilles séparées.

La superposition satisfaisante pour les quatre motifs principaux d'arcs radiés apparait sans surprise, bien que le travail d'identification soit gêné par l'usure profonde sur la pierre. Les différences avec le relevé de 1981 vont plutôt se faire sur des tracés surnuméraires et sur des absences de tracés. Les premiers relèvent des accidents naturels dans la pierre, que les techniques actuelles parviennent à bien différencier des tracés anthropiques. Les seconds sont dus aux limites de l'acuité visuelle humaine qui ne peut identifier certains enlèvements trop fugaces mais qui, surtout, ne permet pas aux humains de mémoriser les différents états d'une surface éclairée selon plusieurs incidences successives. Ce sont finalement ces manques qui font la grande différence, car ces tracés renseignent désormais une autre composition où chaque signe est fondamental à l'heure d'entamer un récit sur la scène. Ce qui pourrait passer pour un détail sans grande conséquence peut s'avérer comme un complément indispensable à la bonne perception des motifs et à leur interprétation.

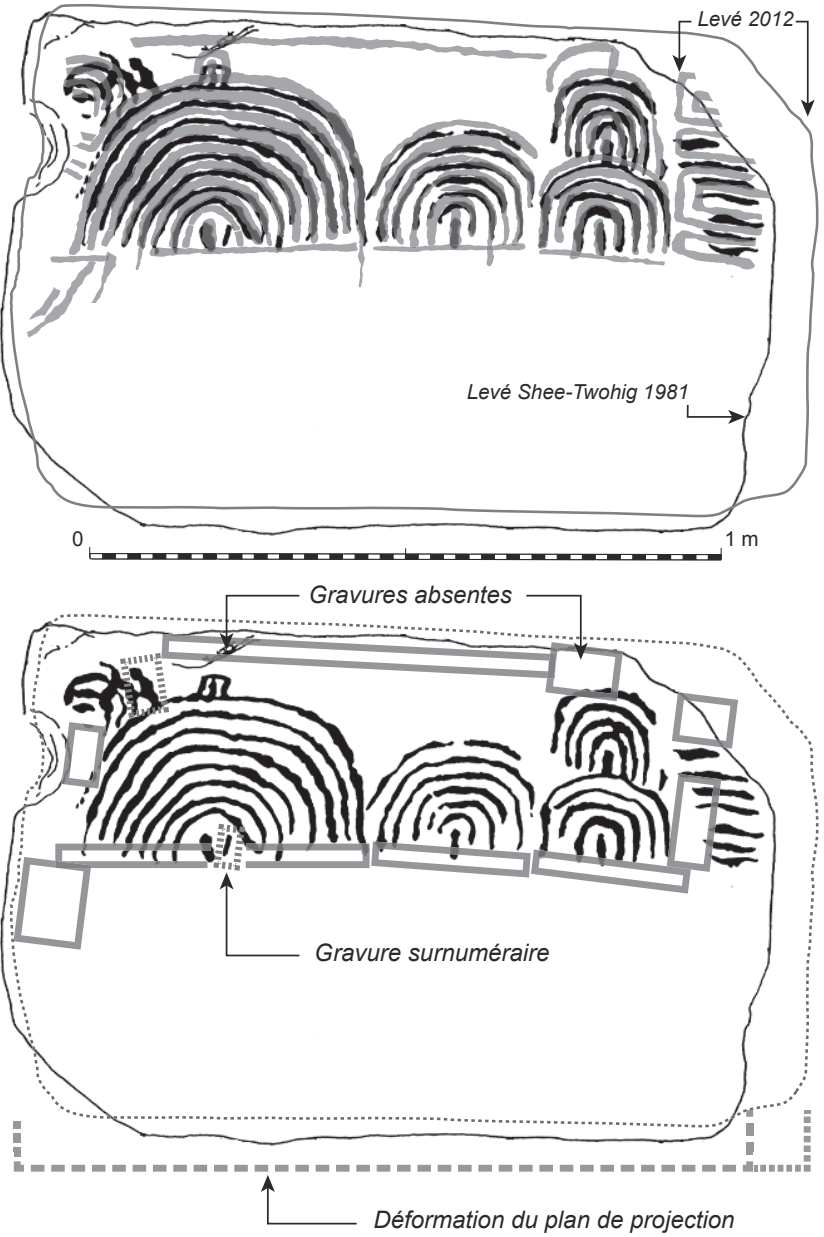

Figure 15 : Comparaison signe à signe par tracés superposés : synthèse Shee-Twohig, 1981 et résultats actuels sur la face supérieure de S12.

Figure 15: Comparison sign to sign by superimposed lines: synthesis by Shee-Twohig 1981 and current results on the superior face of S12.

\section{LA POSSIBILITÉ D'UNE CHRONIQUE}

Il n'est pas fréquent que l'archéologue du Néolithique et des architectures monumentales puisse travailler dans un musée lapidaire dans des conditions de laboratoire. Les observations poussées, aidées par la fine résolution des enregistrements tridimensionnels, permettent en effet la remontée d'événements le plus souvent illisibles sur le terrain.

\section{Biographie d'un monolithe : cassures, fractures, dressages, usures}

La possibilité d'observer les sections entières des deux extrémités tronquées de $\$ 12$ était une opportunité de choix dans ce processus d'enregistrement, d'analyse et de représen- 
tation. Sachant que deux bords du monolithe sont naturels et conservés comme tels, les deux extrémités ne pouvaient être que réduites afin de se conformer à la dimension du couloir, l'ajustement étant fait au centimètre près. Cette intervention sur les tranches de la dalle a bien été notée par nos collègues ayant œuvré à son enlèvement avant l'introduction d'un moulage en ciment (Le Roux, 1981, 1982).

Cette opportunité est moins anodine qu'il n'y parait. On sait combien le débitage volontaire a pu s'inscrire dans les hypothèses des archéologues constatant des réemplois de dalles gravées dans certains monuments funéraires morbihannais du IVe millénaire (Minot, 1964; L'Helgouac'h, 1983 et de nombreux auteurs à la suite). Au-delà des aspects anthropologiques contenus dans les assertions et les commentaires (iconoclastie, etc.), la démonstration scientifique de ce débit de carrier pêchait par l'absence de preuves concrètes ou d'argumentaire construit, a fortiori sur des monolithes aussi considérables que le Grand Menhir de Locmariaquer sur lequel aucune trace de débitage n'est actuellement visible qui pourrait témoigner en faveur de ces actions (Bougis, 1994; Boujot, Cassen, 2000).

\section{Tranche 3}

La section 3 est entièrement travaillée par percussion, probablement plus par volonté de faire disparaitre des aspérités que d'obtenir une surface préparée en vue d'une exposition ou d'une implantation de gravure (fig. 16). On suppose, en effet, qu'une partie de la dalle était invisible de par son insertion dans le sol néolithique (mais un effet de "marche " était possible en avant de la dalle de pavage de la chambre, laissant une certaine lisibilité de la tranche 1). C'est donc bien l'ajustement du monolithe aux dimensions du couloir qui a motivé cette intervention au Néolithique.

La numérisation de l'objet a aussitôt fait ressortir une énorme encoche assez régulière obtenue par percussion répétée depuis la surface supérieure de la pierre, venant confirmer l'observation faite par C.-T. Le Roux (1982). Car l'encoche est en réalité un demi-cylindre évasé en surface, preuve d'un enlèvement de matière depuis cette face dorsale en vue d'un creusement progressif du granite.

Une première hypothèse pouvant expliquer ce travail et cette morphologie doit envisager un appareillage de bois monté à l'entrée de la chambre qui aurait pu s'appuyer sur cet enfoncement (par exemple : tenon d'une porte en bois dans une demi mortaise). Mais un second demi-cratère s'observe aux côtés du premier, moins développé, plus tronqué, tandis que la face inférieure présente deux autres encoches mais sans que leurs centres ne coïncident parfaitement avec les deux précédents. Prises dans un premier temps pour deux nouvelles hémi-mortaises, une inspection plus attentive révélait qu'elles n'étaient ni taillées ni piquetées (fig. 17). Seules

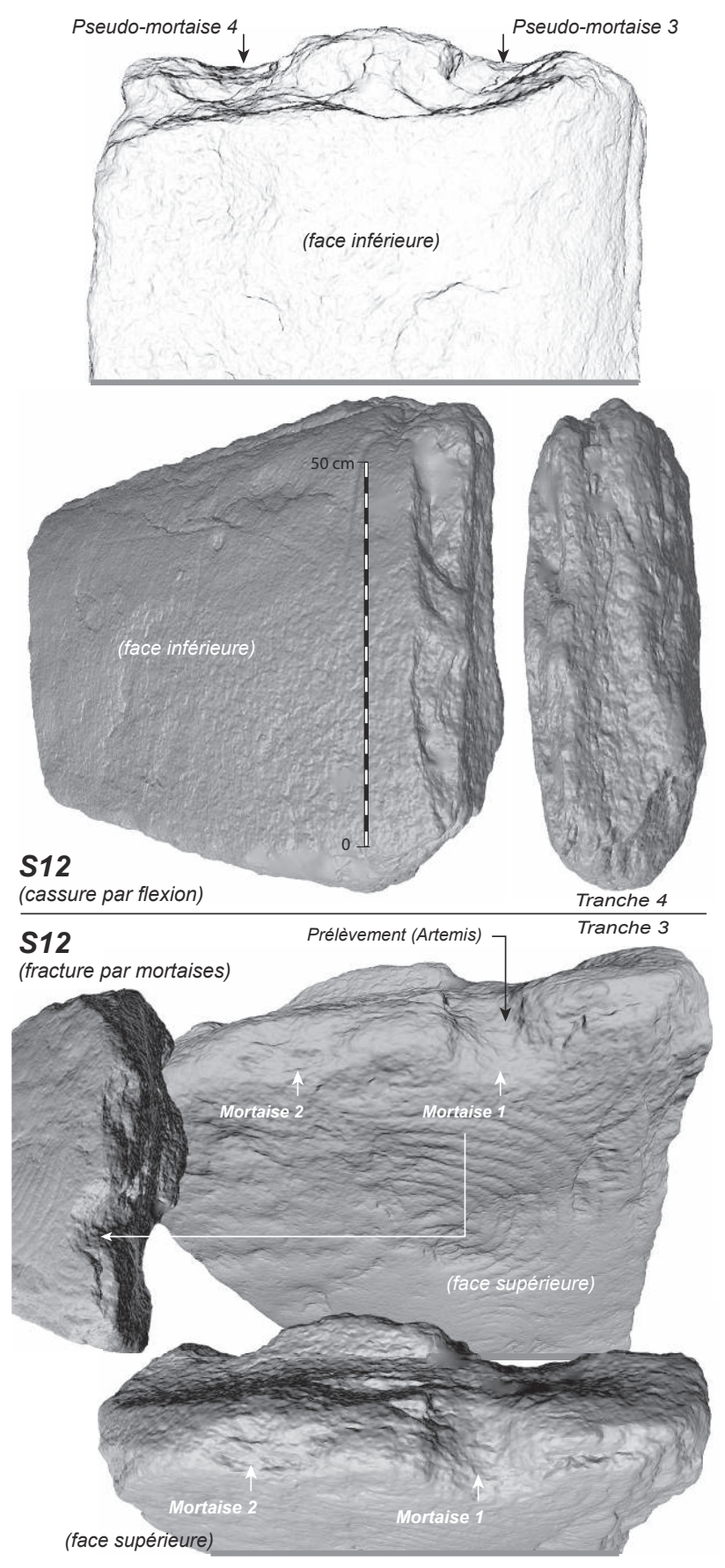

Figure 16 : Représentation des tranches 4 et 3 à partir du modèle 3D.

Figure 16: Representation of sections 4 and 3 from the 3D model.

les précédentes anomalies sont bien des mortaises creusées en vue d'une fracture contrôlée de la pierre, peut-être par enfoncement de coins (bois gorgé d'eau, pierre ?).

La première encoche très visible a été désignée par mortaise 1, sa voisine sur la face supérieure mortaise 2, et sur la face inférieure en partant du même côté se succèdent les 
Figure 17 : Tranche 3 de la dalle S12. Surface de cassure reprise par piquetage; localisation des restes de mortaises et des pseudo-mortaises; position de l'échantillon de charbon daté, pris dans la roche écrasée (Lyon-10785).

Figure 17: Section 3 of the S12 paving stone. Surface of break taken back by piquetage; location of the rests of mortises and pseudo-mortises; position of the charcoal sample dated, taken in the crushed rock (Lyon-10785).

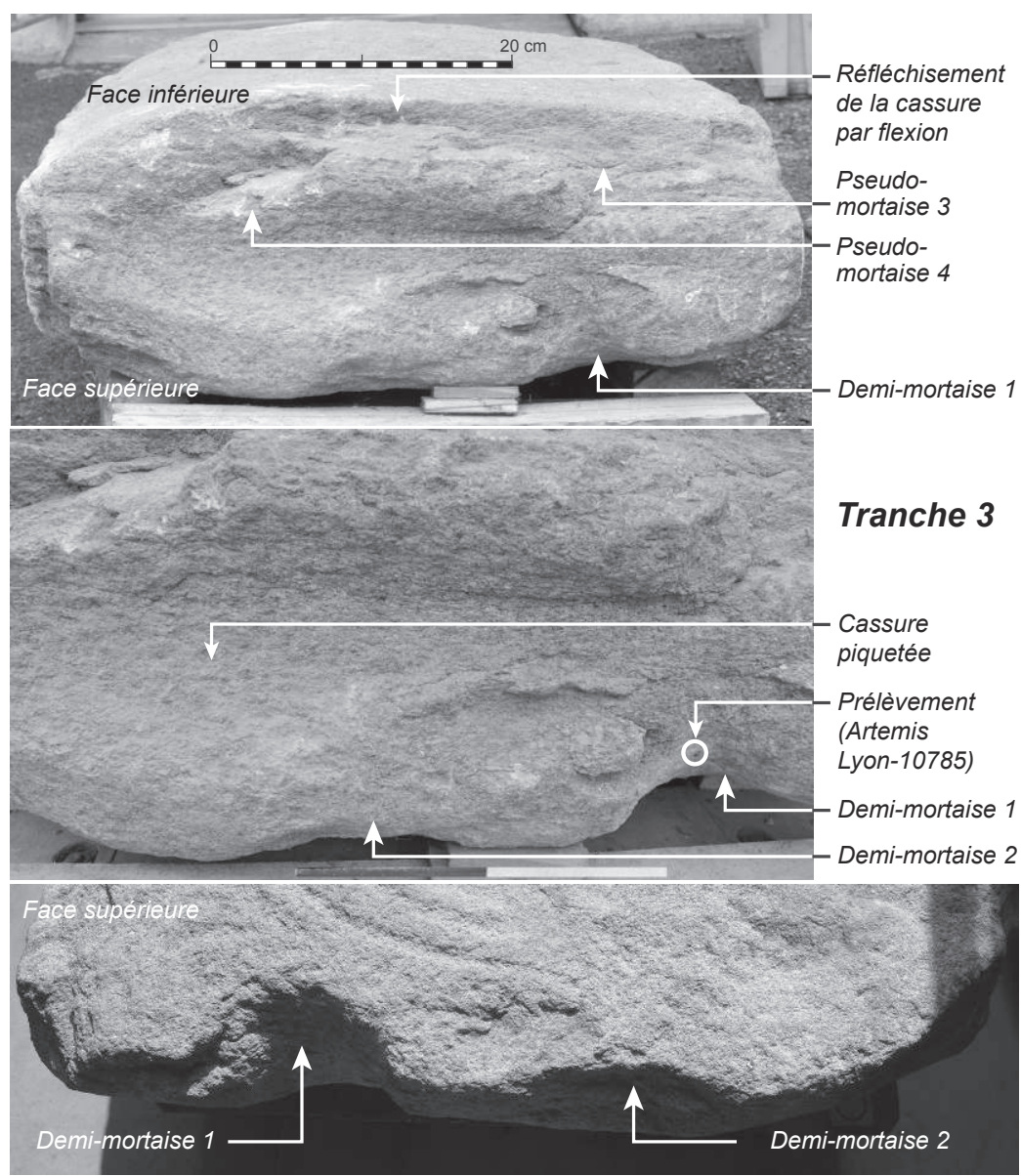

pseudo-mortaises 3 et 4 . La figure 16 résume les différents points de vue permettant de découvrir ces aménagements (ou encoignures - Bessac, 2002), ou ce qu'il en reste après le traitement opéré sur la cassure pour la régulariser. On note cependant que cette cassure conserve un réfléchissement consécutif à la rupture du granite qui n'est peut-être pas le résultat attendu d'un débit par coins gorgés d'eau, où la surface de fracture devrait être plus droite que cela. Il est donc à se demander si un basculement en porte-à-faux, même minime, n'aurait pas provoqué la rupture par flexion suivant les points de faiblesse, occasionnant alors ce réfléchissement caractéristique.

C'est en tout cas la première fois en Morbihan que sont prouvés ces efforts de débitage maîtrisé sur stèles, tous les autres exemples mobilisés pour défendre l'hypothèse - et notamment le cas du Grand Menhir ou la propre dalle de couverture de Gavrinis - ne présentant que des surfaces droites très jointives caractéristiques de cassures " naturelles " (chutes au sol des monolithes); la numérisation récente de ces surfaces n'a d'ailleurs fait que confirmer ces absences d'encoignures et de boîtes de débitage.

\section{Tranche 4}

La tranche 4 présente, au contraire de T3, une rupture nette et rectiligne, perpendiculaire à l'axe longitudinal de la dalle (fig. 16). Seul un angle a subi un enlèvement, probablement par taille (négatif bien visible d'au moins un grand éclat enlevé par percussion).

Cette section offre par ailleurs la forme caractéristique d'une fracture par flexion, dans sa variante " en charnière " où se note un réfléchissement bien connu des lithiciens (Pelegrin, O'Farrell, 2005). Ajoutons que cette cassure a été entièrement reprise par piquetage pour réduire les arêtes afin de ramener le relief à une ligne plus ou moins perpendiculaire aux bords, et peut-être en s'ajustant aux contraintes de largeur à l'emplacement prévu pour le dépôt du pavage. Ce type de rupture nous semble consécutif à une chute du monolithe originellement érigé, à l'image du dernier fragment du Grand Menhir qui présente ce même réfléchissement (Cassen [dir.], 2009). 


\section{Une datation ${ }^{14} \mathrm{C}$ directe sur $\mathrm{S} 12$}

La dalle S12, nous l'avons vu, était un monolithe dont le motif primitif ne pouvait être «lu » qu'en position verticale, autrement dit une ancienne stèle qui fut exactement calibrée pour entrer dans la largeur voulue du couloir. L'examen rapproché des états de surface, d'une diversité remarquable (en termes de fracture, percement, mise en forme, dressage), a conduit à l'observation de plusieurs mouchetures noires incrustées dans la partie écrasée du granite, notamment dans la mortaise 1; un grattage de la surface sur $3 \mathrm{~cm}^{2}$ a permis à cet endroit de récolter un produit d'altération du granite dans lequel trois charbons (de chêne) en paillettes furent prélevés sous loupe binoculaire et adressé au laboratoire du radiocarbone à Lyon pour tenter une datation AMS.

Le résultat positif (Lyon-10785: $5305 \pm 35$ BP [-4241, $-4005 \mathrm{BC}]$ ) est donc très encourageant. Il date probablement la première phase de construction du cairn de Gavrinis, au moment de l'introduction des dalles. Quand les conditions s'y prêtent, voici par conséquent un nouveau moyen d'intervention et d'enquête sur des éléments d'architecture.

\section{Une datation ${ }^{14} \mathrm{C}$ indirecte sur $\mathrm{S} 11$}

Le nettoyage de l'intérieur du couloir, en vue du levé photogrammétrique du sol, donna l'occasion de travailler au contact de la dalle de pavage S11, voisine de S12. Une fois nettoyée (raclée, brossée) et débarrassée des sédiments modernes accumulés par la fréquentation touristique, S11 dévoila une lentille concave de sédiment jaunâtre, préservée au creux du monolithe en granite (fig. 18). Un charbon bien visible apparaissant en surface, le limon argileux fut extrait, tamisé et trois autres charbons furent recueillis et analysés. Deux taxons ont été identifiés, Pomoïdée de type poirier/pommier et chêne. Les deux charbons de Pomoïdée de plus petit diamètre $(27 \mathrm{~mm})$ ont été sélectionnés pour la datation. Considérant la croissance radiale mesurée (2,71 mm/an), l'âge des bois dont sont issus ces charbons peut être estimé à environ cinq ans, ce qui permet d'éviter l'effet « vieux bois » et ainsi obtenir une date au plus près de l'occupation du site.

La date AMS obtenue par l'intermédiaire du programme Artemis (Lyon-10787 : $5065 \pm 40$ BP [-3964, -3773 BC]) donne ainsi un premier aperçu de la fréquentation de l'intérieur de la tombe à couloir (Cassen et al., 2014b); elle est exactement contemporaine de l'occupation de la façade de la Table des Marchands (Locmariaquer - Cassen et al., 2009). D'un ouvrage de stèles antérieur à ce dolmen provient, on le sait, le fragment d'une dalle ayant servi à couvrir la chambre de Gavrinis (Le Roux, 1984).

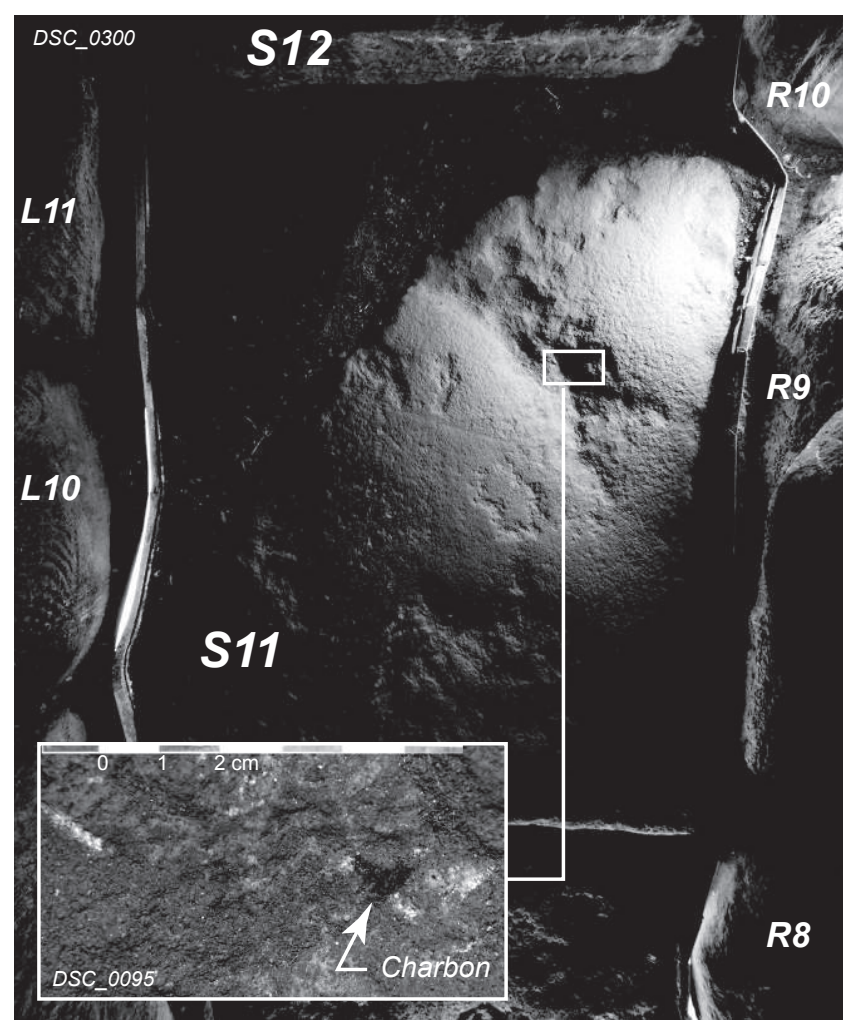

Figure 18 : (Voir planche couleur VI) Charbon visible dans un limon argileux jaunâtre piégé au fond d'une dépression sur S11 (Lyon-10787).

Figure 18: (See colour plate VI) Visible charcoal in a yellowish clayey silt trapped at the bottom of a depression on S11 (Lyon-10787).

Gavrinis n'avait jusqu'ici été daté que par un seul résultat radiocarbone (Le Roux, 1983). Un blocage pierreux important était en effet reconnu pour avoir condamné l'entrée du dolmen en oblitérant la façade; un amoncellement de moellons qui pourrait être également le résultat des éboulis du cairn (Cassen et al., 2013b ; Grimaud, 2015). La date fournie par un des pieux carbonisés dans cet amas (GIF 5766 : $4470 \pm 80 \mathrm{BP}[-3480,-2950 \mathrm{BC}])$ indique que cette possible " condamnation " de la tombe, ou éboulis principal en façade, a dû intervenir au Néolithique récent.

\section{Conclusions}

La dalle $S 12$ est un monolithe tronqué à ses deux extrémités et gravé sur ses deux faces (supérieure ou dorsale, et inférieure ou ventrale) ainsi que sur ses deux tranches naturellement planes. Ce bloc de granite est un probable fragment de stèle, manifestement en réemploi, disposé comme pavage de seuil entre couloir et chambre. Cette position au sol, à l'image des dalles voisines dans la structure d'accès, 
a provoqué une usure considérable de la surface, notamment sur les points hauts du relief (poli apparent, gravures effacées).

Profitant d'une conservation privilégiée au sein d'une collection lapidaire, l'enregistrement des surfaces a été conjointement mené en lasergrammétrie, photogrammétrie et clichés numériques sous éclairages tournants. Ces trois techniques se complètent parfaitement et renseignent des manques qui, en l'absence de ces corrections, ajouts ou soustractions, nous auraient induit en erreur quant à l'inventaire des signes et au descriptif des états de surface.

Grâce aux modèles 3D, un tableau comparé des volumes théoriques obtenus, et donc des masses calculées, permet d'évaluer la pertinence d'une estimation de ce type faite à partie de la lasergrammétrie et de la photogrammétrie (programmes 123Catch et Photoscan, la solution Visual SFM devant être abandonnée étant donné l'exigence scientifique de l'opération) (tableau 2). En considérant que la densité du granite est de 2,7 (valeur maximale des précis de géologie), le poids de la dalle est estimé à $589 \mathrm{~kg}$ avec le modèle lasergrammétrique, alors que le modèle issu d'123DCatch en 2012 le porte à $607 \mathrm{~kg}$, puis à $580 \mathrm{~kg}$ en 2016, tandis que Photoscan indique $599 \mathrm{~kg}$. L'écart moyen entre ces différentes mesures n'excède pas $10 \mathrm{~kg}$.

Confrontées à la masse réellement mesurée au dynamomètre, qu'il s'agisse des données pointues de la lasergrammétrie ou de celles a priori moins précises de la photogrammétrie, toutes semblent avoir tendance à surestimer les quantités, sans pour autant que ce défaut condamne la méthode, l'erreur générée étant acceptable. Cette différence est expliquée par la masse volumique du granite communément indiquée qui n'est pas exactement celle de la roche carnacoise : le ratio issu du volume de notre modèle de référence et de la pesée au dynamomètre indique en effet une densité de 2,592 (et non 2,7).

La manipulation virtuelle du bloc permet également de bien renseigner certains détails des opérations techniques l'ayant affecté. Les extrémités de $\$ 12$ se distinguent en effet, l'une par l'aspect naturel de la cassure par flexion, l'autre par l'intervention humaine visible à travers deux demi boîtes de débitage qui ont aidé, guidé, la rupture dans le granite selon un plan perpendiculaire à l'axe longitudinal de la dalle. On ne peut dire si ces mortaises ont permis l'introduction de coins en bois gorgés d'eau en vue de l'éclatement de la roche, ou si leur seule présence permettait après un choc en porte-à-faux de fracturer la dalle dans le sens souhaité. Le résultat est cependant assez précis pour satisfaire l'insertion $\mathrm{du}$ monolithe aux dimensions du couloir, au centimètre près, aidé en cela par une reprise par percussion directe des arêtes des cassures pour en réduire le relief.

Quant aux gravures, détectables à travers les modèles tridimensionnels, elles ne rendent leur information la plus pertinente qu'après le traitement à la tablette graphique des photographies compilées des surfaces. Ces compositions gravées font appel à la même technique (tracés en creux) faces supérieure et inférieure, mais sont distinctes en termes de géométrie et de représentation.

- La face ventrale, invisible au temps du fonctionnement de la tombe, réunit un motif que nous qualifions de phallique, explicite selon nos critères interprétatifs, sans les euphémismes habituellement rencontrés sur les motifs régionaux ("boucles » latérales); il est peut-être à ce titre un des plus anciens de la séquence morbihannaise; il est aussi largement effacé par un martelage important du granite, qui ne laisse pas d'intriguer.

- La face supérieure est couverte sur une moitié de son étendue par plusieurs motifs d'arcs radiés ainsi que par des signes quadrangulaires emboîtés, manifestement dans le "style » de Gavrinis. Comme sur la face inférieure, plusieurs tracés sont cependant tronqués par la retaille du monolithe d'origine. Les relations d'antéro-postériorité, restituées par les éclairages tournants, permettent d'assurer une modélisation chronologique : une division de l'espace est tout d'abord opérée au moyen de deux rectilignes parallèles, entre

\begin{tabular}{|l|c|c|c|}
\hline Technique de mesure & Volume $\left(\mathrm{m}^{3}\right)$ & Masse $(\mathrm{kg}) ;$ densité $=2,7$ & Masse $(\mathrm{kg}) ;$ densité $=2,592$ \\
\hline Lasergrammétrie & 0,218 & 589 & - \\
\hline 123DCatch 2012 & 0,225 & 607 & 583 \\
\hline 123DCatch 2016 & 0,215 & 580 & 557 \\
\hline Agisoft Photoscan V. 1.2.4. & 0,222 & 599 & 575 \\
\hline Pesée au dynamomètre & & 565 & \\
\hline
\end{tabular}

Tableau 2 : Tableau comparé des deux techniques (lasergrammétrie et photogrammétrie) mises en ouvre pour enregistrer le volume et calculer la masse du monolithe S12, au regard du poids effectif mesuré au peson. La densité du granite $(\mathrm{d}=2,592)$ a été calculé à partir du volume donné par le nuage de points (lasergrammétrie).

Table 2: Compared board of both techniques (lasergrammetry and photogrammetry) implemented to record the volume and to calculate the mass of the monolith S12, with regard to the actual weight measured by the dynamometer. The density of the granite $(d=2,592)$ was calculated from the volume extracted from the laser point cloud. 
lesquelles vont s'inscrire, de la gauche vers la droite (dans la configuration actuelle du bloc disposé dans le couloir), plusieurs motifs d'arcs radiés. L'interprétation du geste, du déroulé et des figures n'est bien entendu pas l'objet de la présente étude.

- Sur les tranches latérales, offrant des surfaces planes en raison d'épontes ou miroirs de faille, deux registres similaires en nature s'opposent néanmoins par la géométrie : composés de segments obliques, l'un est fait de " triangles " emboîtés, l'autre de "triangles » opposés par les sommets.

Les surfaces entièrement couvertes des orthostates de Gavrinis marquent cependant la limite opérationnelle de la méthode dite des éclairages tournants. Faute de recul sur les bords de ces supports, l'information se perd (secteurs surexposés à la prise de vue) et la précision escomptée ne peut pas être atteinte. C'est pour cette raison que le recours aux éclairages virtuels maîtrisés s'avère l'étape majeure d'un développement technique que nous appelons de nos vœux, alors que ces ombrages artificiels ne sont pas encore capables de rivaliser - à échelle d'acquisition comparable - avec les photographies sous illumination rasante et tournante. Dans cette perspective, le principe des protocoles actuels ne devrait pas être modifié pour autant.

L'observation attentive des surfaces des monolithes, ayant subi fractures et mise en forme, pourrait aussi s'avérer une nouvelle voie de recherche quand la préservation d'éléments organiques le permet. S12, pour une part "naturellement " cassée, est aussi fracturée et calibrée à la fin du V $V^{\mathrm{e}}$ millénaire, tandis que la dalle voisine $S 11$, qui piégeait encore matière à datations par le radiocarbone, témoigne d'une fréquentation du couloir au tout début du IV e millénaire. Une datation plus précise du site, et en particulier des différentes temporalités liées aux interventions sur les orthostates gravés, sur les dalles de couvertures et sur les pierres de pavage, est l'autre objectif accompagnant bien entendu la recherche relative à la structure et au contenu du programme iconographique. En dehors des signes gravés aux dos de certains piliers, plusieurs décors dans le style de Gavrinis sont en effet invisibles (loin sous la surface du pavage); d'autres ont été tronqués pour adapter le support à la morphologie de la construction; d'autres encore ont été réalisés après la mise en place de certains orthostates lors de l'allongement vraisemblable $\mathrm{du}$ couloir. L'investigation en cours tente par conséquent de mieux comprendre cette longue durée d'utilisation et de compréhension (ou de mécompréhension, ou de rejet par les sociétés néolithiques) des scènes symboliques figurées.

\section{Remerciements}

Nous tenons à remercier tous les collègues ayant contribué à cette investigation: C. Boujot (DRAC Bretagne), qui a recherché et trouvé l'empreinte faite sur la dalle de seuil en vue de son moulage; O. Agogué pour être intervenu avec son service (SDAM, Vannes) lors de la manutention de la dalle; J.-M. Rousset (École centrale, Nantes) pour avoir dirigé la pesée du monolithe; C. Oberlin (Laboratoire du Radiocarbone, Lyon) pour avoir facilité le suivi des échantillons; C.-T. Le Roux pour avoir retracé avec nous l'historique du moulage et pour avoir corrigé une première version de ce texte; $Y$. Belenfant (Compagnie des Ports, Larmor-Baden) qui nous a toujours assuré de sa collaboration sur le site de Gavrinis; C. Obeltz pour nous avoir aidé lors du nettoyage $d u$ sol du monument; C. Rellán Rodríguez pour la traduction du résumé en anglais. Ce programme de recherche a été financé par la DRAC Bretagne et le conseil départemental du Morbihan, avec les moyens techniques et humains du Laboratoire de recherches archéologiques et architectures de l'université de Nantes, de l'École nationale supérieure d'architecture de Nantes et de l'École centrale de Nantes.

\section{Bibliographie}

Bessac J.-C., 2002 - "Carrières antiques de la Gaule. Une recherche polymorphe. Glossaire des termes techniques ", Gallia, 59, p. 189-194.

Boujot C. et Cassen, S., 2000 - "Tertres et pierres dressées ", Éléments d'architecture (Exploration d'un tertre funéraire à Lannec er Gadouer, Erdeven, Morbihan. Constructions et reconstructions dans le Néolithique morbihannais. Propositions pour une lecture symbolique), Éditions chauvinoises, Mémoire 19, Chauvigny, p. 181-206.

Bougis F., 1994 - À propos du Grand Menhir brisé de Locmariaquer, Le Bono, Bougis (éd. d'auteur).

Cassen S. (dir.), 2009 - Autour de la Table. Explorations archéologiques et discours savants sur des architectures néolithiques à Locmariaquer, Morbihan (Table des Marchands et Grand Menhir), ACR 2003-2006, Actes du colloque international, Vannes [Morbihan], 5-7 octobre 2007 (université de BretagneSud, campus Le Tohannic), LARA, Université de Nantes, Nantes.

Cassen S., 2012 - "L'objet possédé, sa représentation : mise en contexte général avec stèles et gravures ", PÉTrequin P., CASSEN S., Errera M., Klassen L., Sheridan A. et Pétrequin A.-M. (éd.), Jade. Grandes haches alpines du Néolithique européen. Ve et IVe millénaires av. J.-C., Cahiers de la MSHE C.N. Ledoux, Presses universitaires de Franche-Comté et Centre de recherche archéologique de la Vallée de l'Ain, t. 2, Besançon, p. 1310-1353. 
Cassen S., Lefebvre B. et Merheb M., 2005 - « Mané Rutual en Locmariaquer (Morbihan). Enregistrement de gravures et simulations architecturales dans une tombe à couloir néolithique ", Revue archéologique de l'Ouest, 22, p. 93-124.

Cassen S., Lanos P., Dufresne P., Oberlin C., Delqué-Kolic E. et Le Goffic M., 2009 - "Datations sur site (Table des Marchands, alignement du Grand Menhir, Er Grah) et modélisation chronologique du Néolithique morbihannais ", in Cassen S. (dir.), Autour de la Table. Explorations archéologiques et discours savants sur une architecture néolithique restaurée à Locmariaquer, Morbihan (Table des Marchands et Grand Menhir), Actes du colloque international, Vannes (Morbihan), 5-7 octobre 2007 (Université de Bretagne-Sud, campus Le Tohannic), Université de Nantes, LARA, Nantes, p. 737-768.

Cassen S., Lescop L., Grimaud V., Querré G. et Suner B., $2013 \mathrm{a}$ - «In search of a representation of the Gavrinis megalithic tomb (Morbihan, France). IV Congreso Internacional de arqueolgia e Informatica Grafica, Patrimonio e Innovacion, Sevilla, 20-22 juin 2012 ", Virtual archaeology review, vol. 4, p. 61-63.

Cassen S., Lescop L. et Grimaud V., 2013b - « Pour une critique de la représentation tridimensionnelle des architectures mégalithiques en Europe occidentale. Méthodes et usages actuels ", Annales de Bretagne et des Pays de l'ouest, t. 120, 1, p. 7-31.

Cassen S., Lescop L., Grimaud V. et Robin G., 2014a "Complementarity of acquisition techniques for the documentation of Neolithic engravings: lasergrammetric and photographic recording in Gavrinis passage tomb (Brittany, France) ", Journal of Archaeological Science 45, p. 126-140.

Cassen S., Grimaud V., Lescop L., Marcoux N., Oberlin C. et Querré G., 2014b - « The first radiocarbon dates for the construction and use of the interior of the monument at Gavrinis (Larmor-Baden, France) », PAST, Prehistoric Society 77, p. 1-4. [http://www.prehistoricsociety.org/files/PAST_77_for_ web.pdf.

Cassen S., Grimaud V., Lescop L. et Lagrost L., 2017 - «Les compositions gravées en Bourgogne ", in Gauthier E. et PÉTREQuin P. (dir.), Objets-signes et interprétations sociales des jades alpins dans l'Europe néolithique, Cahiers de la MSHE C.N. Ledoux n ${ }^{\circ}$ 17, Presses universitaires de FrancheComté, Besançon, p. 846-882.

Closmadeuc G. (de), 1864 - Lîle de Gavrinis et son monument, Vannes, Imp. Lamarzelle.

Closmadeuc G. (de), 1873 - Sculptures lapidaires et signes gravés des dolmens dans le Morbihan. Vannes, Imp. De Lamarzelle.

Closmadeuc G. (de), 1884 - "Gavrinis. Fouilles et découvertes récentes ", Bulletin Société Polymatique du Morbihan, 2, p. 180187.

DAG, 1875-1923. Dictionnaire archéologique de la Gaule: époque celtique, France, Volume 1. Collection de documents inédits sur l'histoire de France, Commission de la topographie des Gaules. Paris, Imprimerie nationale.

Déchelette J., 1912. Une Nouvelle interprétation des gravures de Newgrange et de Gavrinis. L'Anthropologie, XXIII, p. 29-52.

Douau F., 1984 - «Abel Maître, mouleur, restaurateur ", Antiquités Nationales, p. 16-17.

Grimaud V., 2015 - Réexplorer et valoriser les architectures funéraires monumentales du Néolithique de l'Ouest de la FranceÉtude du cairn de Gavrinis, Thèse de doctorat, université de Nantes, école doctorale : Sociétés, Cultures, Echanges, UFR Histoire, Histoire de l'art et archéologie (Laboratoire de recherches archéologiques, UMR 6566), 2 vol.

Kergal (Association archéologique), 1977 - L'allée couverte de Gavrinis : les pierres gravées, Études et travaux n ${ }^{\circ} 2$.

Le Roux C.-T., 1981 - "Bretagne ", Gallia Préhistoire, 24, 2, p. 395-423.

LE Roux C.-T., 1982 - « Nouvelles gravures à Gavrinis, LarmorBaden (Morbihan) ", Bulletin de la Société préhistorique française, t. 79, 3, p. 89-96.

LE Roux C.-T., 1983 - " Larmor-Baden. Tumulus de Gavrinis », Gallia Préhistoire, 26, 2, p. 309-333.

LE Roux C.-T., 1984 - «À propos des fouilles de Gavrinis (Morbihan) : nouvelles données sur l'art mégalithique armoricain ", Bulletin de la Société préhistorique française, 81, 8, p. 240-245.

Lescop L., Cassen S. et Grimaud V., 2013 - " Gavrinis. The raising of digital stones ", in Alonzo C. Addison, Livio De Luca, Gabriele Guidi, Sofia Pescarin (éd.), 2013 Digital Heritage International Congress (28 oct $-1^{\mathrm{er}}$ nov 2013, Marseille, France), CAA Fall 2013 Symposium, Communicating Archaelogy: Theory \& Practice, vol. 2, p. 561-568.

L'Helgouac' ' J., 1983 - «Les Idoles qu'on abat », Bulletin Société Polymatique du Morbihan, 110, p. 57-68.

Luquet G.-H., 1913 - "Les pétroglyphes de Gav'rinis ", L'Anthropologie de Paris, XXIV, p. 153-169.

Maître A., 1885 - "Le Tumulus de Gavrinis. Explication de l'origine des dessins sculptés sur les pierres de l'allée couverte ", Revue archéologique, p. 1-11.

Minoт R., 1964 - «Sur les Gravures mégalithiques du Morbihan », Bulletin Société Polymatique du Morbihan, p. 89-98.

Pelegrin J. et O’Farrell M., 2005 - «Les lamelles retouchées ou utilisées de castanet ", in "Productions lamellaires attribuées à l'Aurignacien : Chaînes opératoires et perspectives technoculturelles XIV congrès de l'UISPP, Liège 2-8 Septembre 2001 ", ArchéoLogiques 1 Luxembourg, p. 103-121.

Péquart M., Péquart St-J. et Le Rouzic Z., 1927 - Corpus des signes gravés des monuments mégalithiques du Morbihan, Paris, Éd. A. Picard.

Roвın G., 2009 - L'Architecture des signes. L'art pariétal des tombeaux néolithiques autour de la mer d'Irlande, Rennes, PUR. 
Shee Twohig E., 1981 - The Megalithic Art of western Europe, Oxford, Clarendon Press.

Stockis E., 1921 - « Les Pétroglyphes de Gavrinis et leur interprétation ", L'Anthropologie XXXI, p. 1-36.

Vourc'h M., Chaigneau C. et Cassen S., 2014 - "Archéologie expérimentale à Gavrinis : vers une compréhension du geste gravé (campagne 2012-2013) ", Journée du "CReAAH " Archéologie, Archéosciences, Histoire, Université de Rennes 1, Rennes, p. 33-38.

\section{Webographie}

123DCatch : [http://www.123dapp.com/catch].

VSFM : [http://ccwu.me/vsfm/README].

Wu C., "VisualSFM: A Visual Structure from Motion System », [http://ccwu.me/vsfm/, 2011].

Wu C., Agarwal S., Curless B. et Seitz S.M., "Multicore Bundle Adjustment ", CVPR 2011.

Agisoft Photoscan, [http://www.agisoft.com/].

Zusammenfassung: Die Untersuchung einer monolithischen Stele - Die Steinplatte S12 auf dem Boden des Grabmonumentes von Gavrinis (Larmor-Baden, Morbihan, Frankreich) - Die Untersuchung einer monolithischen Steinplatte aus einem der berühmtesten Ganggräber des Gebietes von Carnac beschäftigt sich mit methodischen Fragestellungen wie der Dokumentation der gut erhaltenen oder verwitterten Einritzungen sowie mit der detaillierten Morphologie des ausgewählten Gesteins, die beide zur Lesbarkeit und Interpretation der dargestellten Zeichen beitragen können. Verschiedene moderne computergestützte Messmethoden wurden getestet und gestatten einen Vergleich der verschiedenen Ergebnisse, insbesondere bezüglich der Dimensionen, der Volumina und der Massen. Schließlich hat die unerwartete Erhaltung von Holzkohlefragmenten in zwei Rissen der gebrochenen Randbereiche der Steinplatte erlaubt, eine 14C-Datierung vorzunehmen. Diese Datierung hat es gestattet, die absolute Chronologie des Grabmonumentes zu präzisieren, was bis dahin für diese Megalithanlage unmöglich war.

Schlüsselwörter: Wiederverwendete Stele, Neolithikum, Lasergrammetrie, Photogrammetrie, kompilierte Bildsequenzen. 